\title{
Chiral Pyridine-3,5-bis- (L-phenylalaninyl-L-leucinyl) Schiff Base Peptides as Potential Anticancer Agents: Design, Synthesis, and Molecular Docking Studies Targeting Lactate Dehydrogenase-A
}

\author{
Abd El-Galil E. Amr ${ }^{1,2}$, Randa E. Abdel Mageid ${ }^{3}$, Mohamed El-Naggar ${ }^{4}{ }^{\oplus}$, \\ Ahmed M. Naglah 1,5 ${ }^{(D}$, Eman S. Nossier ${ }^{6}$ and Elsayed A. Elsayed ${ }^{7,8, * \mathbb{D}}$ \\ 1 Drug Exploration \& Development Chair (DEDC), Pharmaceutical Chemistry Department, College of \\ Pharmacy, King Saud University, Riyadh 11451, Saudi Arabia; aamr@ksu.edu.sa (A.E.-G.E.A.); \\ amnaglah@gmail.com (A.M.N.) \\ 2 Applied Organic Chemistry Department, National Research Center, Cairo, Dokki 12622, Egypt \\ 3 Photochemistry Department, National Research Center, Cairo, Dokki 12622, Egypt; \\ randaabdelmagid@yahoo.com \\ 4 Chemistry Department, Faculty of Sciences, University of Sharjah, Sharjah 27272, UAE; \\ m5elnaggar@yahoo.com \\ 5 Department of Peptide Chemistry, National Research Centre, Cairo 12622, Egypt \\ 6 Pharmaceutical Medicinal Chemistry Department, Faculty of Pharmacy (Girls), Al-Azhar University, \\ Cairo 12622, Egypt; dr.emannossier@gmail.com \\ 7 Zoology Department, Bioproducts Research Chair, Faculty of Science, King Saud University, Riyadh 11451, \\ Saudi Arabia \\ 8 Chemistry of Natural and Microbial Products Department, National Research Centre, Cairo, \\ Dokki 12622, Egypt \\ * Correspondence: eaelsayed@ksu.edu.sa; Tel.: +966-55-895-3623
}

Academic Editor: Simona Collina Received: 3 February 2020; Accepted: 27 February 2020; Published: 29 February 2020

\begin{abstract}
A series of branched tetrapeptide Schiff bases 3-6 were designed and synthesized from corresponding tetrapeptide hydrazide 2 as a starting material.In vitroevaluation of the synthesized compounds 4-6 against breast MCF-7 carcinoma cells identified their excellent anticancer potency, with $\mathrm{IC}_{50}$ ranging from $8.12 \pm 0.14$ to $17.55 \pm 0.27 \mu \mathrm{M}$ in comparison with the references, cisplatin and milaplatin $\left(\mathrm{IC}_{50}=13.34 \pm 0.11\right.$ and $18.43 \pm 0.13 \mu \mathrm{M}$, respectively). Furthermore, all derivatives demonstrated promising activity upon evaluation of theirin vitroandin vivosuppression of p53 ubiquitination and inhibition assessment for LDHA kinase. Finally, molecular docking studies were performed to predict the possible binding features of the potent derivatives within the ATP pocket of LDHA in an attempt to get a lead for developing a more potent LDHA inhibitor with anti-proliferative potency.
\end{abstract}

Keywords: amino acids; tetrapeptides; Schiff bases; anticancer evaluation; p53 ubiquitination; LDHA; molecular docking

\section{Introduction}

Peptides are short linear chains of amino acids (usually less than 50 AA in length) and are often stabilized by disulfide bonds [1]. Recently, there has been a noticeable increase in the number of publications covering the potential effects of peptides on lipid metabolism and blood pressure (BP), in addition to their antimicrobial, anti-inflammatory, analgesic, antioxidant, immunomodulatory, and anticancer activities [2-4]. 
Still, cancer is one of the most prominent causes of death in developed countries and there is a great demand for the discovery of new chemotherapies with more selectivity for cancer cells and less side effects $[4,5]$. The anticancer effects of peptides have been extensively explored with a raised number of approved peptide-based drugs [6-8]. The great potential of their antitumor activity has been exhibited through the action on multiple molecular pathways like enzymes involved in carcinogenesis with no genotoxicity [9].

Moreover, tumor-targeting peptides are effective alternative entities for the selective delivery of high doses of chemotherapeutic drugs or diagnostic agents to tumor sites while sparing normal tissues. Several peptide hormones have already been applied for tumor targeting, for example, the cyclic octapeptide analogue of somatostatin, octreotide, has been utilized for radiotargeting in neuroendocrine tumor [10]. Additionally, the linear peptide analogue of Luteinizing hormone-releasing hormone (LHRH), AN-152, has been used to target the LHRH receptor of breast cancer, ovarian cancer, and prostate cancer [11]. Recently, most peptidopyridine derivatives that have been synthesized consist of a wide range of pharmaceutical activities, including anticancer, analgesic, anti-convulsant, antiparkinsonian, analgesic, anti-inflammatory, and antimicrobial activities [12-15].

Schiff bases have been important candidates owing to their coordination chemistry, and they can be synthesized and used as ligands in building complex with different metal ions. On the other hand, Schiff bases have been interested with a broad spectrum of biological and pharmacological activities, such as antibacterial, DNA binding, anti HIV, Largicidal, antifertility, anticancer, and anti-inflammatory activities [16-23]. The broad-spectrum biological activities of Schiff bases have been attributed to the $-\mathrm{C}=\mathrm{N}$ - imine bond, where the electrophilic carbon and nucleophilic nitrogen provide excellent binding characters with different nucleophiles and electrophiles, resulting in the inhibition of targeted diseases, enzymes, or DNA replication [24].

The lactate dehydrogenase $(\mathrm{LDH})$ is one of the most prominent proteins and is responsible for the interconversion of lactate and pyruvate, parallel with the interconversion of $\mathrm{NAD}^{+}$and NADH. LDH is present in humans as three subunits-LDHA, LDHB, and LDHC [25]. It is clear that cancer cells mainly produce energy through glycolysis and LDHA is the final enzyme involved in this pathway [26]. As LDHA is a vital supporter of glucose metabolism in cancer cells and its increased level is a marker for many tumors, LDHA can be defined as an anticancer drug target [27]. Previous literatures reported that V-shape structures illustrate good LDHA inhibition, so molecular modification of the potentially identified LDHA inhibitors A-C (Figure 1) might provide more effective hits [28-30].

In the present investigation and as a continuation of our previous work [31-38] in peptide and heterocyclic chemistry, chiral N3,N5-bis[(L-phenylalaninyl-L-leucinyl) substituted aryl or heterocyclic Schiff base]pyridine candidates were successfully designed, synthesized, and plausibly characterized by analytical techniques including IR, nuclear magnetic resonance (NMR), and mass spectroscopy evidences. In addition, their cytotoxicity effects on MCF-7 cancer cell lines, in vivo and in vitro inhibition of p53 ubiquitination, and lactate dehydrogenase-A were demonstrated in detail. Furthermore, molecular docking illustrates the binding affinity to LDHA kinase, which could facilitate the discovery of novel anticancer and LDHA inhibitory agents. 


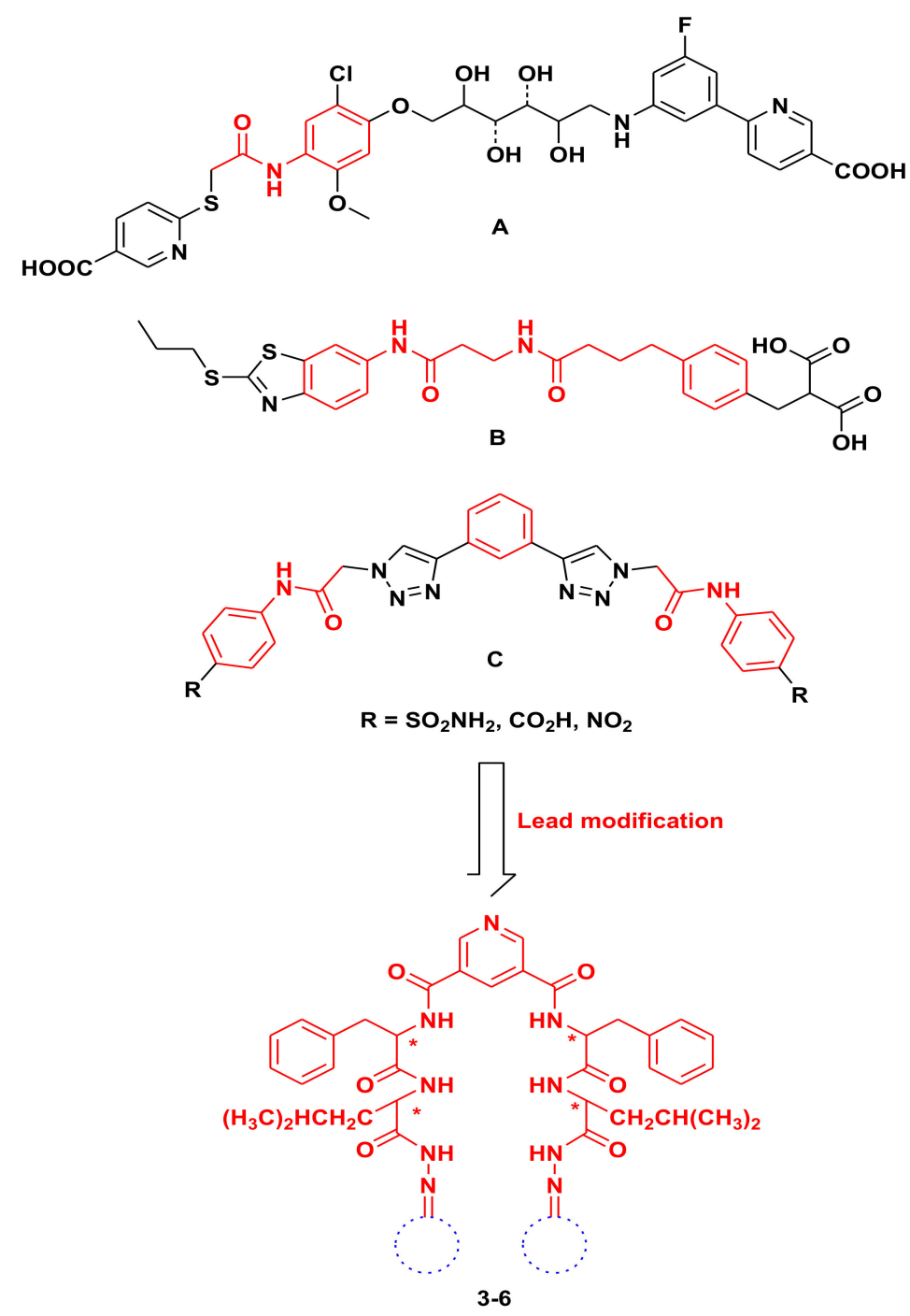

Figure 1. Design of new target derivatives 3-6 via lead modification of known candidates A-C having promising cytotoxic and LDHA inhibitory activities.

\section{Results and Discussion}

\subsection{Chemistry}

In the present work, some of the prepared tetrapeptide derivatives 3-6 were obtained from $N^{\alpha}$-dipicolinoyl-bis[(L-phenylalanyl-L-leucine)hydrazide] 2 , which was obtained from corresponding ester 1 [39]. Reaction of hydrazide 2 with cycloalkanone derivatives in refluxing glacial acetic acid afforded the corresponding $N^{\alpha}$-dipicolinoyl-bis[(L-phenylalanyl-L-leucine)$\mathrm{N}^{\prime}$-cycloalkylidenehydrazide] 3a-d. Condensation of 2 with active carbonyl derivatives, namely, acetylpyridine or substituted acetophenone derivatives in refluxing acetic acid, gave the corresponding $N^{\alpha}$-dipicolinoyl-bis[(L-phenylalanyl-L-leucine)- $\mathrm{N}^{\prime}$-(1-(pyridyl)ethylidene)benzohydrazide] $\mathbf{4 a - c}$ and $N^{\alpha}$-dipicolinoyl-bis-[(L-phenylalanyl-L-leucine)- $\mathrm{N}^{\prime}$-(1-(substituted phenyl) ethylidene)benzohydrazide] $5 \mathbf{a}-\mathbf{e}$, respectively. Finally, treatment of heterocyclic aldehydes in refluxing ethanol gave the corresponding $N^{\alpha}$-dipicolinoylbis-[(L-phenylalanyl-L-leucine)-N'-(1- (furyl- $6 \mathrm{a}$ and thienyl)ethylidene)benzohydrazide] $\mathbf{6 b}$, respectvely (Scheme 1). 


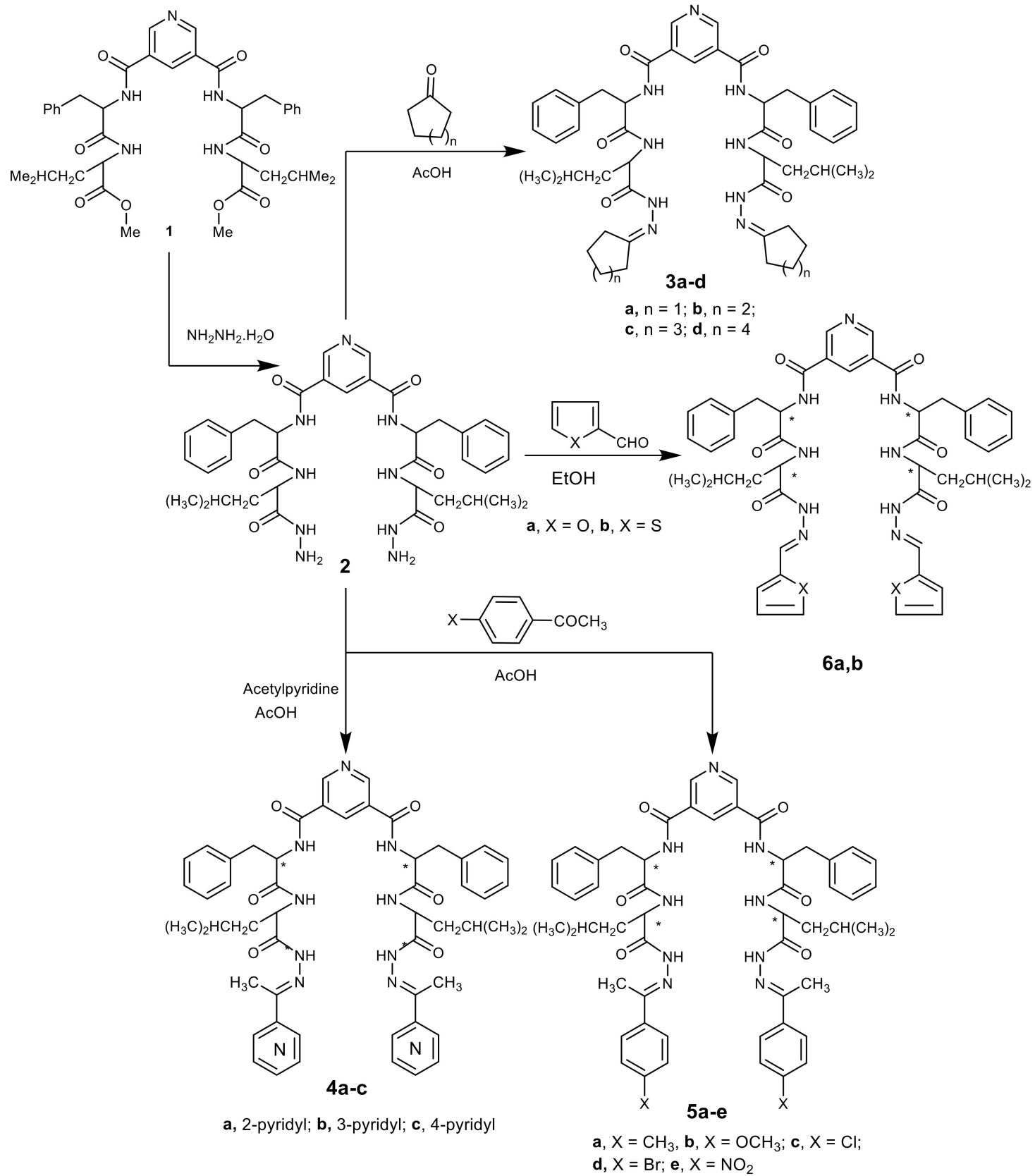

Scheme 1. Synthetic routes for compounds $3 a-d, 4 a-c, 5 a-e$, and $6 a, b$.

\subsection{Biological Evaluation}

\subsubsection{Evaluation of In vitro Anticancer Potentials Against Breast Cancer}

Following the synthesis of the new bis-dipeptide derivatives, we evaluated the anticancer potentials of these derivatives in vitro against breast cancer cells (MCF-7) as well as normal non tumorigenic MCF-10A cells. The obtained results were expressed as $\mathrm{IC}_{50}$ values in $\mu \mathrm{M}$ and were also compared for both cell lines with positive reference controls; that is cisplatin and milaplatin. The obtained results are presented in Figure 2. Generally, it can be seen that our newly synthesized bis-dipeptides have potential anticancer activities against MCF-7 cells. Furthermore, the obtained $\mathrm{IC}_{50}$ values ranged from $8.12 \pm 0.14$ to $17.55 \pm 0.27 \mu \mathrm{M}$ for compounds $4 \mathbf{b}$ and $5 \mathbf{a}$, respectively. Additionally, all compounds were much active than milaplatin $\left(\mathrm{IC}_{50}=18.43 \pm 0.13 \mu \mathrm{M}\right)$. Similarly, except for $5 \mathbf{a}, 5 \mathbf{b}$, 
and 6a, all other compounds were more active than cisplatin $\left(\mathrm{IC}_{50}=13.34 \pm 0.11 \mu \mathrm{M}\right)$. The most active compound (4b) was more active by about $39.1 \%$ and $55.9 \%$ than cisplatin and milaplatin, respectively.

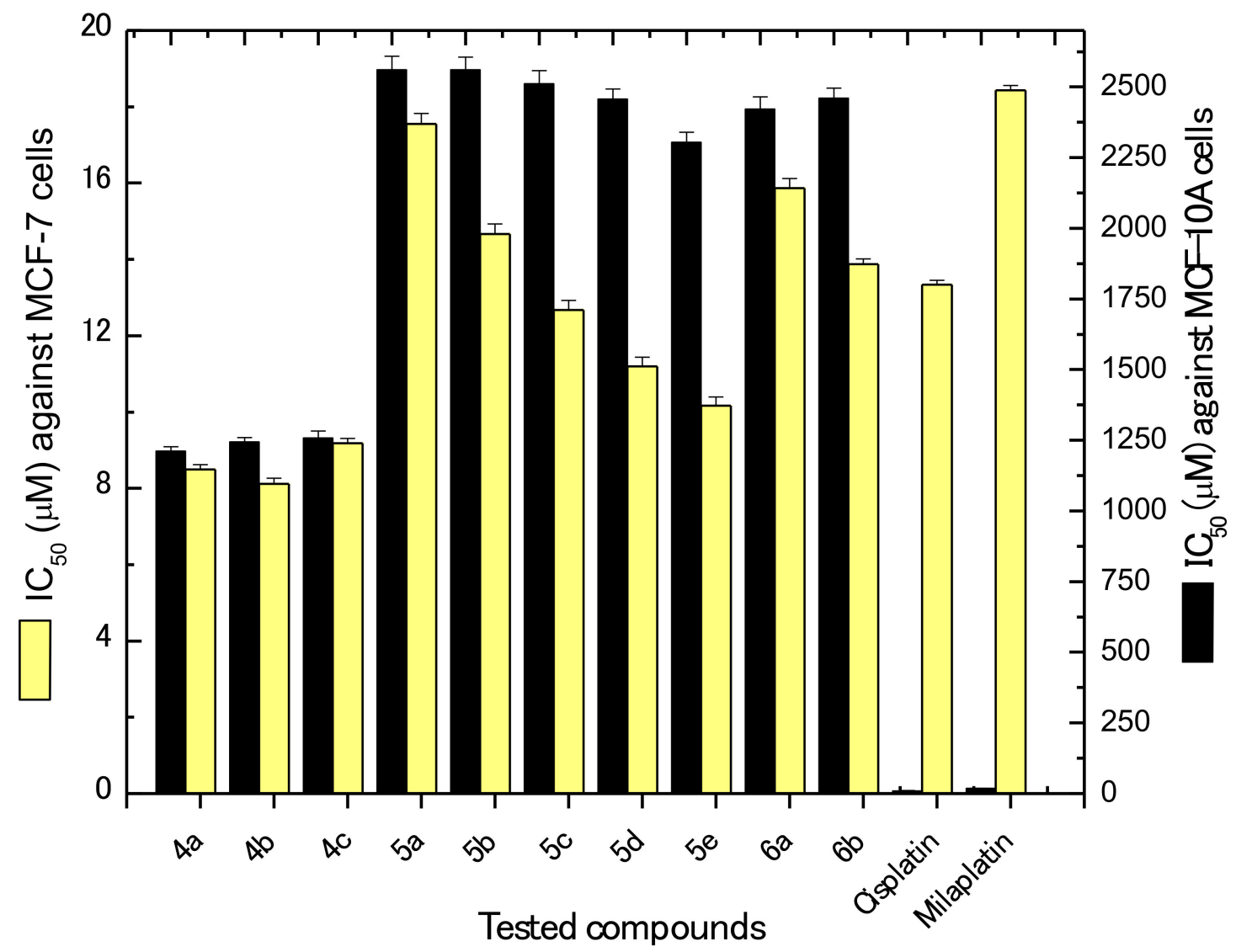

Figure 2. In vitro cytotoxic activities of the tested compounds $\mathbf{4}-\mathbf{6}$ against MCF-7 and MCF-10A cell lines.

Generally, the anticancer activities of the prepared compounds can be arranged in descending order as follows: $4 b>4 a>4 c>5 e>5 d>5 c>6 b>5 b>6 a>5 a$. This arrangement can be related with the structure function relationship. Pyridine containing derivatives $4 \mathbf{a}-\mathbf{c}$ were more active than benzene ring-containing ones $(\mathbf{5 a}-\mathbf{d})$, which were in turn less active than the five-membered ring compounds $(\mathbf{6} \mathbf{a}, \mathbf{b})$. Furthermore, the nitrogen-position in pyridine nucleus affected the activities where the 3-pyridyl $>2$-pyridyl $>4$-pyridyl ones. This may be attributed to the aromaticity and the location of the nitrogen atom in the pyridine ring. On the other hand, substitution on the benzene ring affected the anticancer activity (nitro $5 \mathbf{e}>$ bromo $5 \mathrm{~d}>$ chloro $5 \mathrm{c}>$ methoxyl $5 \mathbf{b}>$ methyl $5 \mathrm{a}$ ), owing to the difference in the electron withdrawing or donation capacity of the substituting group. Finally, the substitution with five-membered rings afforded notably increased activity in the thiophene derivative $\mathbf{6 b}$ than the furan one $\mathbf{6 a}$, owing to the difference in electronegativity between the oxygen and sulfur atoms. Moreover, the larger size of the sulfur atom makes the thiophene ring more similar in size to a phenyl ring and gives it a more pronounced aromatic character, compared with the furan ring, thus more closely resembling the benzene ring of the active compounds. Bis-dipeptide Schiff bases have been previously reported to exhibit anticancer activities owing to their ionophoric properties, which may contribute to apoptosis induction $[40,41]$.

It is worth mentioning that comparing $\mathrm{IC}_{50}$ results between both MCF-7 and non-tumorigenic MCF-10A cells revealed that our newly synthesized compounds are far less toxic against normal cells. The $\mathrm{IC}_{50}$ values obtained for the most and least potent derivatives ( $4 \mathbf{b}$ and $\mathbf{5 a}$, respectively) were higher by about 142.7- and 145.9-fold, respectively, against MCF-10A. It is generally preferable that 
pharmaceutical candidates with potential anticancer properties should exhibit less toxicity against normal cells. The fact that normal cells are usually less affected by possible anticancer candidates may be attributed to the fact that, upon cancer initiation and development, cancer cells overcome normal growth regulation pathways present in normal cells, and thusthey tend to be affected by other different regulatory growth pathways, which are not evident in the case of normal cells.

\subsubsection{In vivo Anti-Breast Cancer}

In this section, we developed a breast cancer animal xenograft model for the evaluation of thein vivo anticancer potential of our newly prepared compounds. We chose the derivatives that showed the lowest $\mathrm{IC}_{50}$ values in the previous in vitro investigation; namely, $\mathbf{4 a}, \mathbf{4 b}, \mathbf{4} \mathbf{c}$, and $\mathbf{5 e}$. The results obtained (Figure 3) showed that all four tested compounds have a great potential to decrease tumor growth in animal model, where the decrease in tumor growth effect increased gradually with the treatment period up to eight days of treatment. At that point, the highest decrease in tumor growth obtained ranged from $81.3 \%$ to $74.2 \%$ for compounds $4 \mathbf{a}$ and $5 \mathbf{e}$, respectively. After wards, the noticeable effects were more or less constant up to 14 days of treatment. However, by the end of the treatment period (20 days), the percentage of tumor growth inhibition decreased to about $75.4 \%, 73.1 \%$, $70.5 \%$, and $60.8 \%$ for compounds $\mathbf{4 a}, \mathbf{4 a}, \mathbf{4}$, and $\mathbf{5 e}$, respectively. Our results are in good agreement with those previously published on the in vivo anticancer potential of Schiff bases owing to apoptosis induction [42].

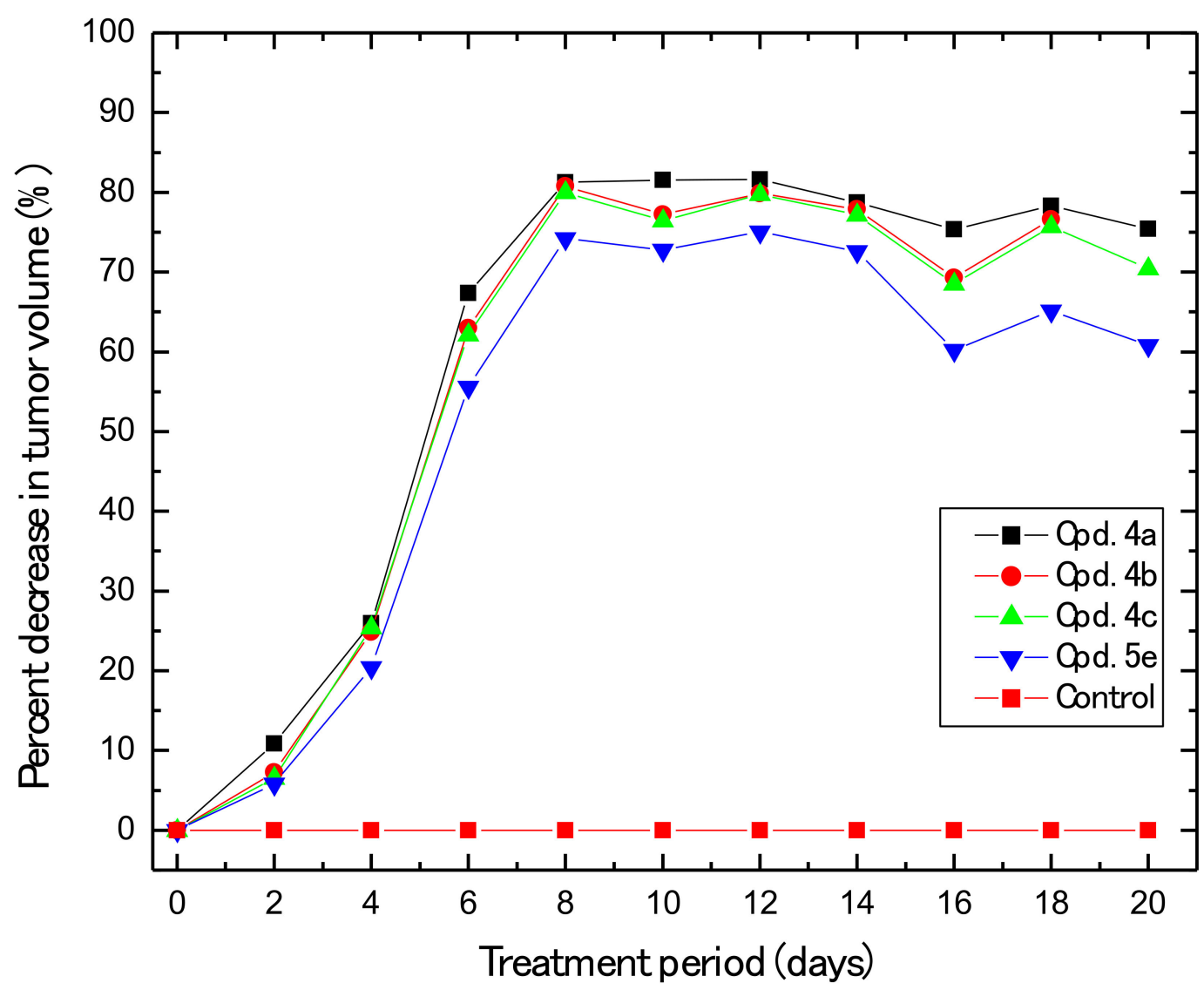

Figure 3. Decreased percentage ofin vivotumor growth in response to different compound treatments.

\subsubsection{In vitro and In vivo Inhibition of p53}

Several cancer prevention drugs depend mainly on the ubiquitination activities against p53 as a suppressor protein. When E3 ubiquitin protein ligase HDM2 binds to p53, the resulting mechanism 
is the inhibition of its ability as a transcription activator. Accordingly, the regulative mechanism is acting negatively. Therefore, blocking the p53 binding site on HDM2 provides for a better possible antitumorigenic molecule. Currently, murine double minute 2 (MDM2) is considered as the regulator of choice when it comes to investigatingp53 inhibition [43].

The results obtained (Figure 4) showed that all prepared derivatives exhibited potential in vitro as well as in vivo suppression of p53 ubiquitination when compared with the positive control (diphenyl imidazole). The obtained in vitro $\mathrm{IC}_{50}$ values ranged from $21.34 \pm 0.13$ to $44.33 \pm 0.34 \mu \mathrm{M}$ for compounds $4 \mathbf{a}$ and $5 \mathbf{a}$, respectively. For thein vivo results, the $\mathrm{IC}_{50}$ values ranged from $0.12 \pm 0.0011$ to $0.31 \pm$ $0.0010 \mu \mathrm{M}$ for compounds $4 \mathbf{a}$ and $\mathbf{6 a}$, respectively. Furthermore, it can be seen that inhibitory effects of 4a were higher by about 12.2- and 15.7-fold compared with the positive control for in vitro and in vivo p53 ubiquitination, respectively.

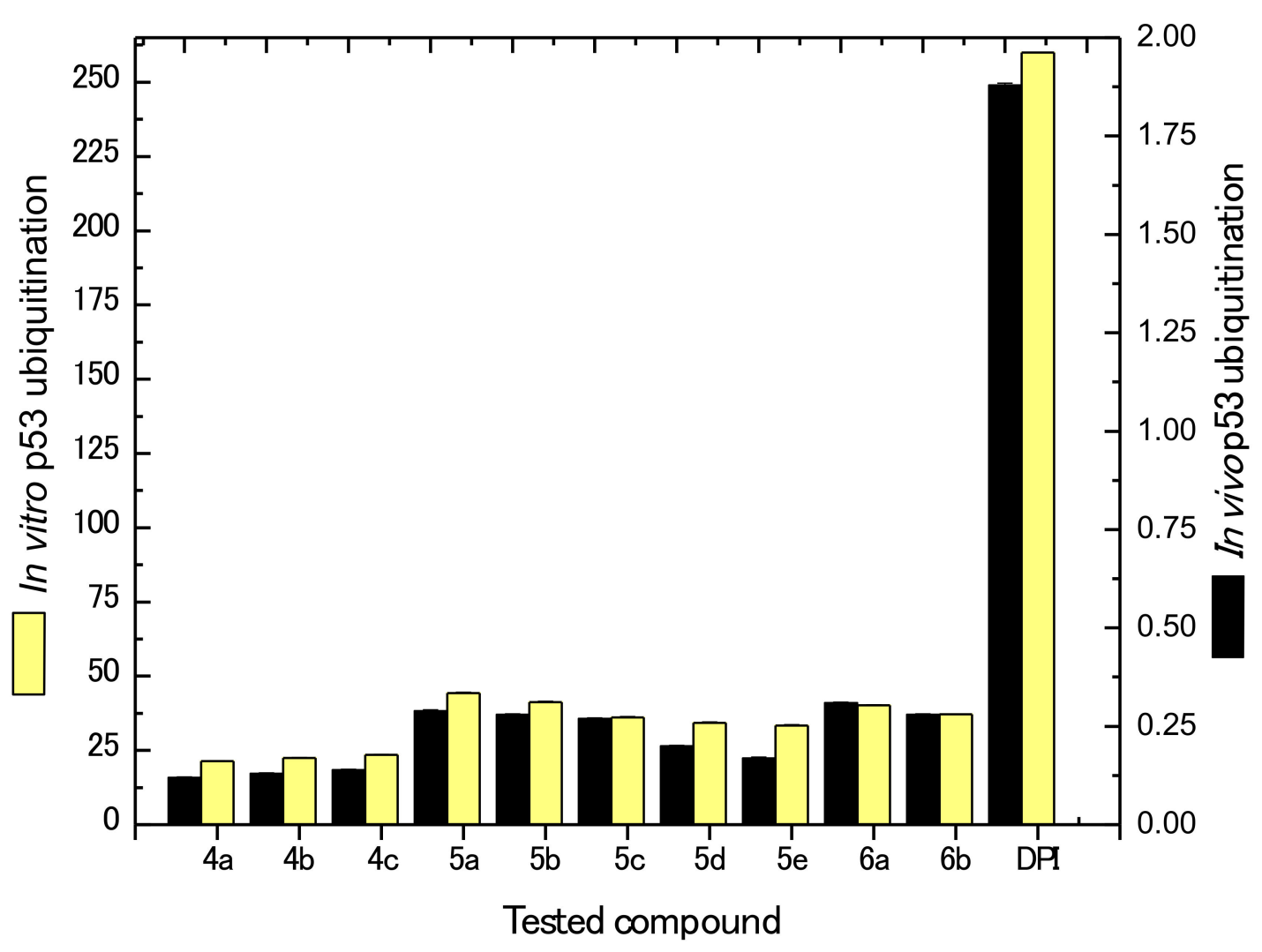

Figure 4. In vitro and in vivop53 ubiquitination of the newly synthesized compounds.

\subsubsection{Kinase Inhibition Studies}

The newly synthesized compounds 4-6 were evaluated for their ability to inhibit LDHA in comparison with galloflavin as a known isoform nonselective LDHA inhibitor [44,45]. The choice of galloflavin as a reference was based on the fact that it is potent anticancer agent and commercially available [46-50]. The results presented in Figure 5 show that all investigated derivatives exhibited better LDHA inhibition compared with galloflalvin, where the $\mathrm{IC}_{50}$ values ranged from $60.54 \pm 0.56$ to $141.56 \pm 0.98 \mu \mathrm{M}$ for compounds $\mathbf{5 c}$ and $\mathbf{5 e}$, respectively. These values correspond to about 2.66 - and 1.14-fold in increase in the inhibitory activity compared with that recorded for galloflavin $\mathrm{IC}_{50}=161.05$ $\pm 0.32 \mu \mathrm{M})$. 


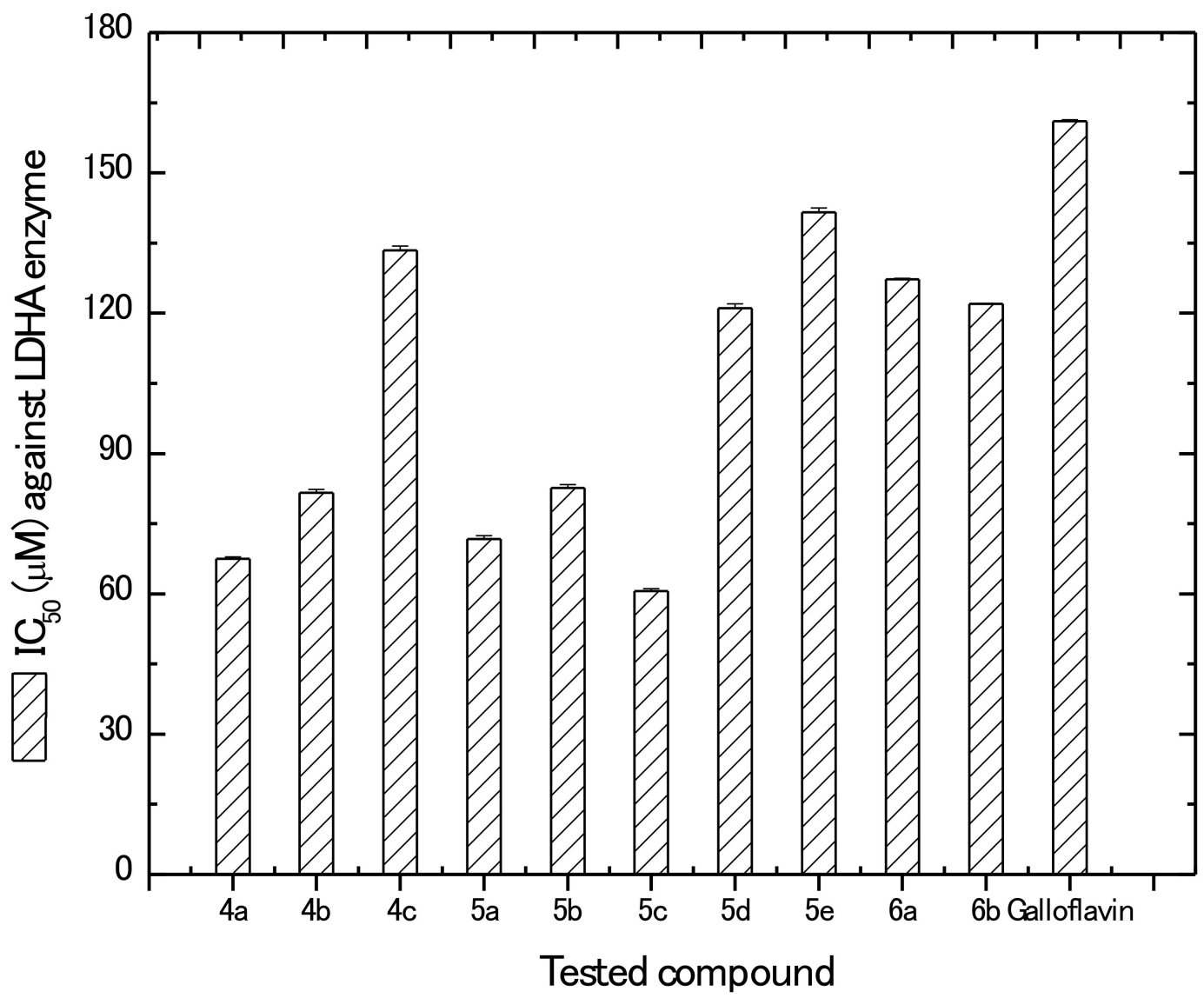

Figure 5. In vitro kinase inhibition activity of the tested compounds 4-6 against human lactate dehydrogenase-A.

\subsection{Molecular Modeling Studies}

In order to further understand and illustrate the interaction of compounds 4-6 with the LDHA active site, the docking modeling studies were done in an open loop conformation of the enzyme. The docking studies were developed using Molecular Operating Environment software, MOE 10.2008 software [51,52] with X-ray crystallographic structure of LDHA (PDB ID: 4ZVV) [53]. The root mean square deviation (RMSD) value of 8.93 was calculated via redocking of the co-crystallized ligand, GNE-140, into the pocket site of LDH-A with docking score energy of $-9.73 \mathrm{kcal} / \mathrm{mol}$. The docking scores of the screened compounds 4-6 were all in the range of -15.60 to $-11.29 \mathrm{kcal} / \mathrm{mol}$. Representations of the docking results of these compounds with LDHA are depicted in Table 1and Figures 6-8. 
Table 1. Molecular docking data of the synthesized compounds 4-6 in comparison with GNE-140 within the active site of LDHA kinase using MOE software version 2008.10.

\begin{tabular}{|c|c|c|c|c|}
\hline Compd. NO. & $\begin{array}{l}\text { Docking Score } \\
\text { (Kcal/mol) }\end{array}$ & $\begin{array}{l}\text { Amino Acid Residues } \\
\text { (Bond Length } \mathrm{A}^{\circ} \text { ) }\end{array}$ & $\begin{array}{l}\text { Atoms of } \\
\text { Compound }\end{array}$ & Type of Bond \\
\hline GNE-140 & -9.73 & $\begin{array}{l}\text { Asn137(2.75); } \\
\text { Arg168(2.99); } \\
\text { His192(2.74) }\end{array}$ & $\begin{array}{l}\mathrm{O}(\mathrm{OH}) ; \\
\mathrm{O}(\mathrm{CO}) ; \\
\mathrm{O}(\mathrm{OH})\end{array}$ & $\begin{array}{l}\text { H- acc } \\
\text { H- acc } \\
\text { H-acc }\end{array}$ \\
\hline $4 a$ & -14.22 & $\begin{array}{c}\text { Asn137(2.54); } \\
\text { Arg168; } \\
\text { His192(2.66) }\end{array}$ & $\begin{array}{c}\mathrm{O}(\mathrm{CONH}) \\
\text { 2-pridyl; } \\
\mathrm{N}(2 \text {-pridyl })\end{array}$ & $\begin{array}{c}\mathrm{H}-\mathrm{acc} \\
\text { Arene-cation } \\
\text { H-acc }\end{array}$ \\
\hline $4 b$ & -13.62 & $\begin{array}{c}\text { Asn137(2.68); } \\
\text { Arg168; } \\
\text { His192(2.88) }\end{array}$ & $\begin{array}{c}\mathrm{O}(\mathrm{CONH}) ; \\
\text { 3-pridyl; } \\
\mathrm{N}(3 \text {-pridyl) }\end{array}$ & $\begin{array}{c}\mathrm{H}-\mathrm{acc} \\
\text { Arene-cation } \\
\text { H-acc }\end{array}$ \\
\hline $4 c$ & -11.29 & $\begin{array}{l}\text { Asn137(2.77); } \\
\text { Arg168 }\end{array}$ & $\begin{array}{l}\mathrm{O}(\mathrm{CONH}) \\
\text { 3-pridyl }\end{array}$ & $\begin{array}{c}\mathrm{H}-\text { acc } \\
\text { Arene-cation }\end{array}$ \\
\hline $5 a$ & -13.01 & $\begin{array}{c}\text { Arg98; } \\
\text { Arg168; } \\
\text { Tyr238(3.20); } \\
\text { Thr247(3.00) }\end{array}$ & $\begin{array}{c}\mathrm{C}_{6} \mathrm{H}_{5}-4-\mathrm{CH}_{3} ; \\
\mathrm{C}_{6} \mathrm{H}_{5}-4-\mathrm{CH}_{3} ; \\
\mathrm{O}(\mathrm{CONH}) ; \\
\mathrm{O}(\mathrm{CONH})\end{array}$ & $\begin{array}{c}\text { Arene-cation } \\
\text { Arene-cation } \\
\text { H-acc } \\
\text { H- acc }\end{array}$ \\
\hline $5 b$ & -12.78 & $\begin{array}{c}\text { Arg168; } \\
\text { His192(2.90) } \\
\text { Tyr238(3.00); }\end{array}$ & $\begin{array}{c}\mathrm{C}_{6} \mathrm{H}_{5}-4-\mathrm{OCH}_{3} \\
\left.\mathrm{O}\left(\mathrm{C}_{6} \mathrm{H}_{5}-4-\mathrm{OCH}\right)_{3}\right) \\
\mathrm{O}(\mathrm{CONH})\end{array}$ & $\begin{array}{c}\text { Arene-cation } \\
\text { H-acc } \\
\text { H- acc }\end{array}$ \\
\hline $5 c$ & -15.60 & $\begin{array}{c}\text { Arg98; } \\
\text { Arg168; } \\
\text { Tyr238(3.06); } \\
\text { Thr247(2.70) }\end{array}$ & $\begin{array}{c}\mathrm{C}_{6} \mathrm{H}_{5}-4-\mathrm{Cl} ; \\
\mathrm{C}_{6} \mathrm{H}_{5}-4-\mathrm{Cl} ; \\
\mathrm{O}(\mathrm{CONH}) ; \mathrm{O}(\mathrm{CONH})\end{array}$ & $\begin{array}{c}\text { Arene-cation } \\
\text { Arene-cation } \\
\text { H-accH- acc }\end{array}$ \\
\hline $5 \mathrm{~d}$ & -11.89 & $\begin{array}{c}\text { Arg98; } \\
\text { Arg168; } \\
\text { Asn137(2.88) }\end{array}$ & $\begin{array}{l}\mathrm{C}_{6} \mathrm{H}_{5}-4-\mathrm{Br} ; \\
\mathrm{C}_{6} \mathrm{H}_{5}-4-\mathrm{Br} ; \\
\mathrm{O}(\mathrm{CONH})\end{array}$ & $\begin{array}{c}\text { Arene-cation } \\
\text { Arene-cation } \\
\text { H-acc }\end{array}$ \\
\hline $5 e$ & -11.37 & $\begin{array}{c}\text { Arg168; } \\
\text { Asn137(3.05) }\end{array}$ & $\begin{array}{l}\mathrm{C}_{6} \mathrm{H}_{5}-4-\mathrm{NO}_{2} \\
\mathrm{O}(\mathrm{CONH})\end{array}$ & $\begin{array}{c}\text { Arene-cation } \\
\text { H-acc }\end{array}$ \\
\hline $6 a$ & -12.06 & $\begin{array}{l}\text { Asn137(2.88); } \\
\text { Tyr238 }\end{array}$ & $\begin{array}{l}\mathrm{O}(\mathrm{CONH}) \\
\text { furan }\end{array}$ & $\begin{array}{c}\mathrm{H}-\mathrm{acc} \\
\text { Arene-cation }\end{array}$ \\
\hline $6 b$ & -12.88 & $\begin{array}{l}\text { Asn137(2.35); } \\
\text { Tyr238 }\end{array}$ & $\begin{array}{l}\mathrm{O}(\mathrm{CONH}) \\
\text { thiophene }\end{array}$ & $\begin{array}{c}\mathrm{H}-\text { acc } \\
\text { Arene-cation }\end{array}$ \\
\hline
\end{tabular}

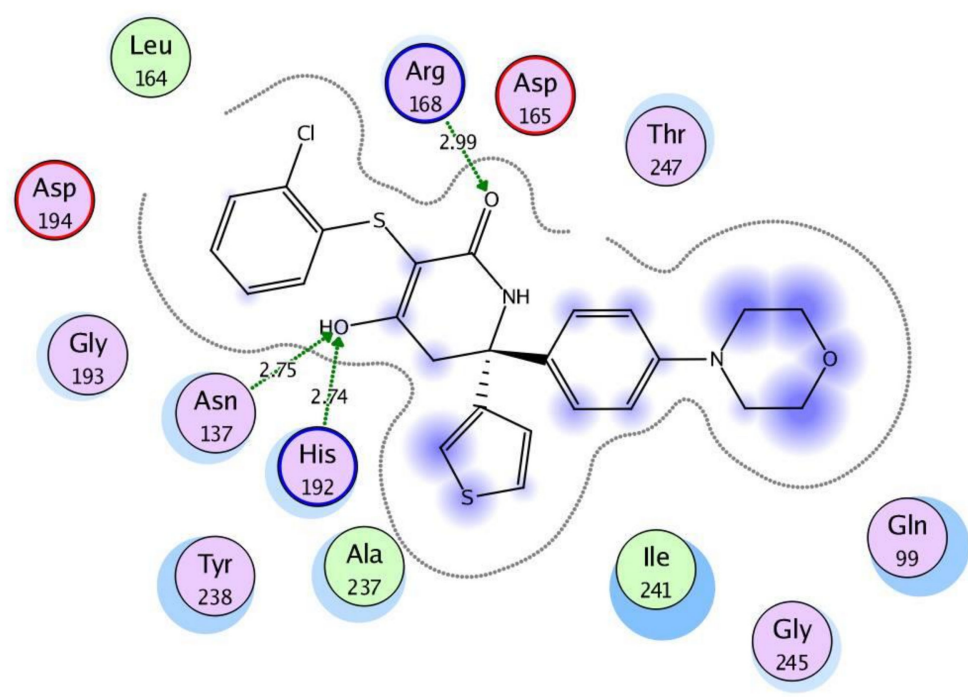

Figure 6. Cont. 


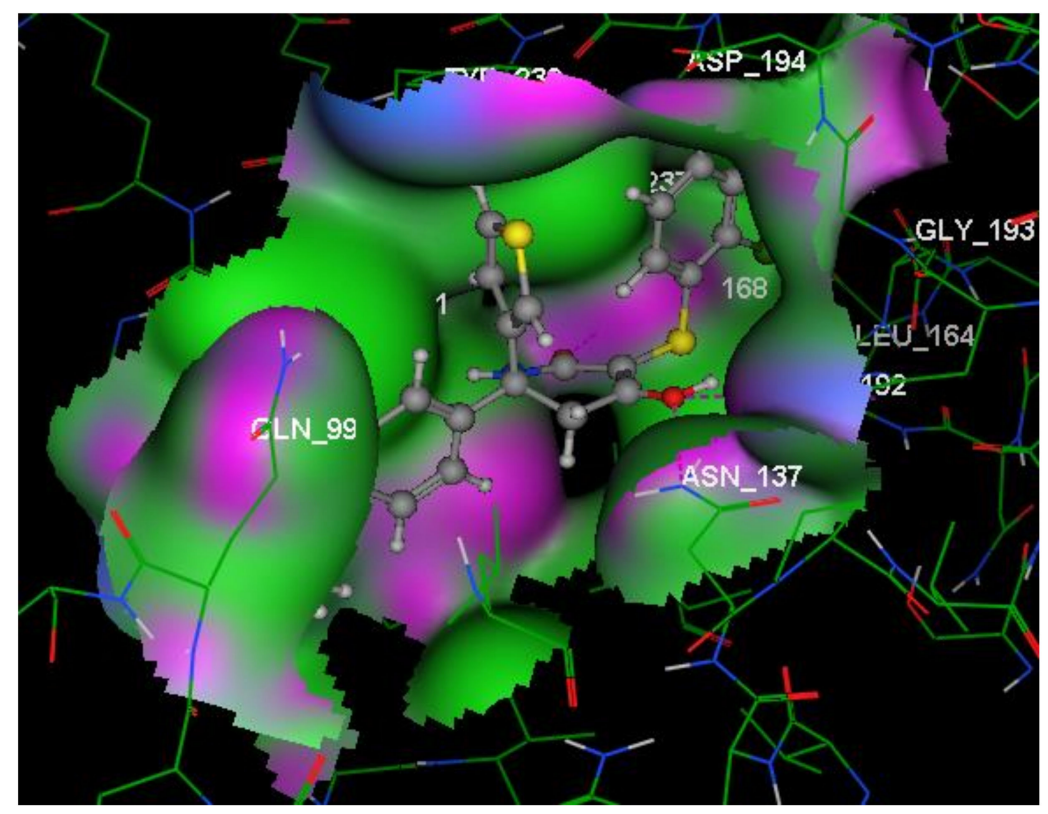

B

Figure 6. Two-dimensional (A) and 3D (B) patternsillustrating the proposed binding mode of the co-crystallized ligand GNE-140 in the LDHA binding pocket (PDB code: 4ZVV). Hydrogen bonding interactions with the protein are shown as dashed lines. Green color reflects a hydrophobic area, pink color reflects a high polar area, and blue color reflects a mild polar area.

As the reported binding mode of GNE-140 with LDHA [53], the oxygen of the hydroxyl group binds to the active site via two hydrogen bond acceptors with the sidechains of Asn137 and His192 (distance: 2.75 and $2.74 \AA$ ), respectively. Additionally, the carbonyl oxygen forms H-bonding with the sidechain of Arg168 (distance: $2.99 \AA$ ) (Figure 6).

The increased inhibitory activity of the newly synthesized derivatives 4-6 against LDHA enzyme could be partially explained by the evidence obtained from the docking studies (Table 1). By focusing on the highly potent inhibitors $4 \mathbf{a}$ and $\mathbf{5 c}$ (Figures 7 and 8), the essential residue Arg168 connected to the newly formed 2-pyridyl (in 4a) and 4-chlorophenyl moieties (in 5c) by arene-cation interactions. Moreover, the compound $4 \mathbf{a}$ was fitted nicely in the active pocket through two hydrogen bond interactions between nitrogen of 2-pyridyl moiety and oxygen of the amide group with the key amino acids His192 and Asn137 (distance: 2.66 and $2.54 \AA$ ), respectively.

The enhanced inhibitory potency of $\mathbf{5 c}$ may be attributed to its stabilization within the ATP binding pocket via further arene-cation interaction between the other 4-chlorophenyl and Arg98, and two H-bond acceptors between the oxygens of the two amide groups located at opposite sites around the parent pyridine ring with the sidechain of Tyr238 and the backbone of Thr247 (distance: 3.06 and $2.70 \AA$ ), respectively. Additionally, the essential amino acids Ala97, Asn137, and Pro138 were located between the two wings of the symmetrical side chains facing the central core (pyridine moiety) (Figure 8). 


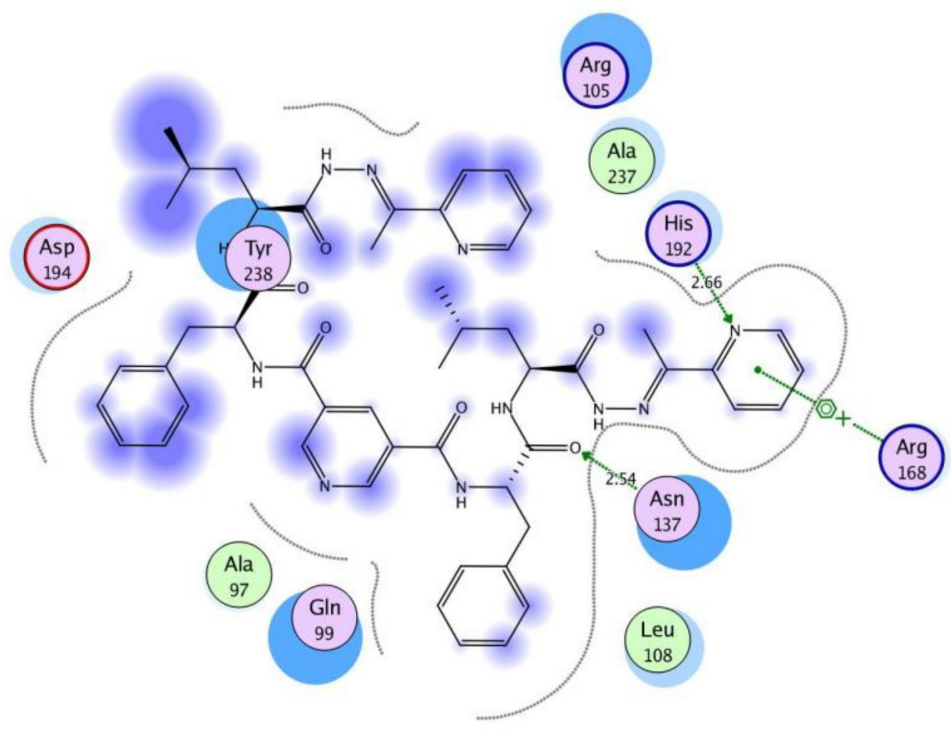

A

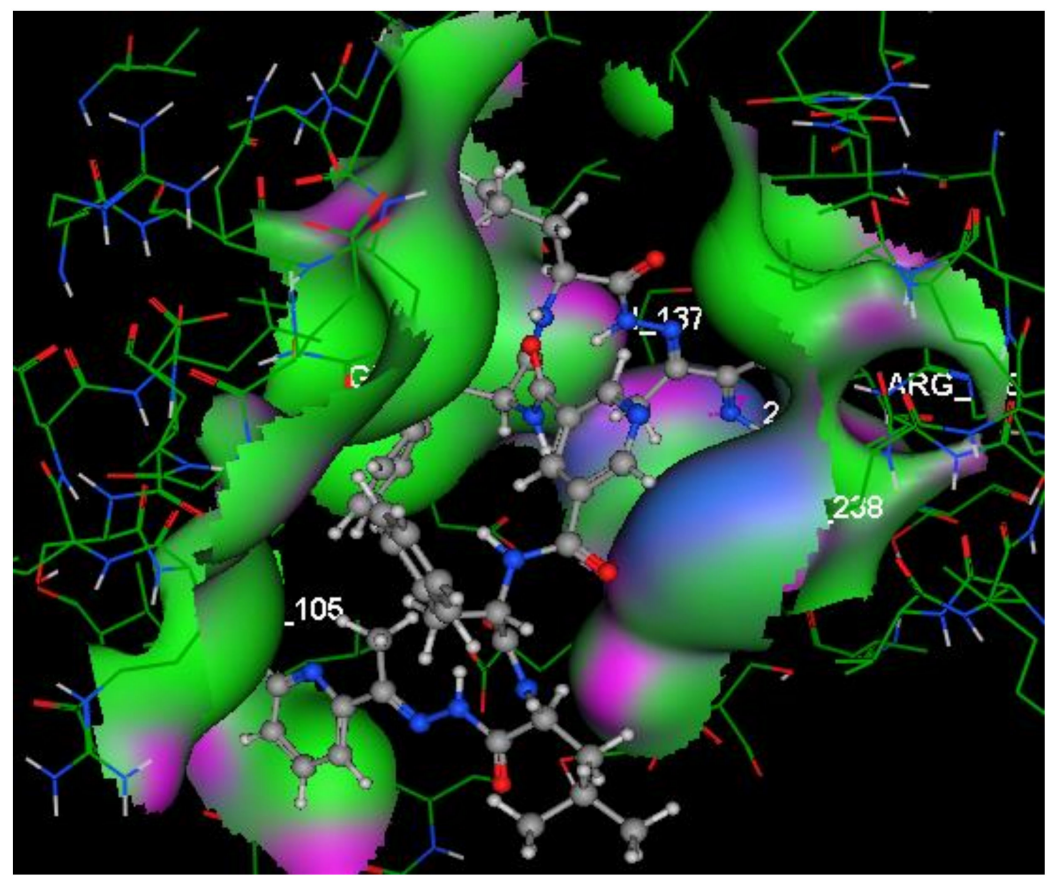

B

Figure 7. Two-dimensional (A) and 3D (B) patternsillustrating the proposed binding mode of the compound $4 \mathbf{a}$ in the LDHA binding pocket (PDB code: 4ZVV). Hydrogen bonding interactions with the protein are shown as dashed lines. Green color reflects a hydrophobic area, pink color reflects a high polar area, and blue color reflects a mild polar area. 


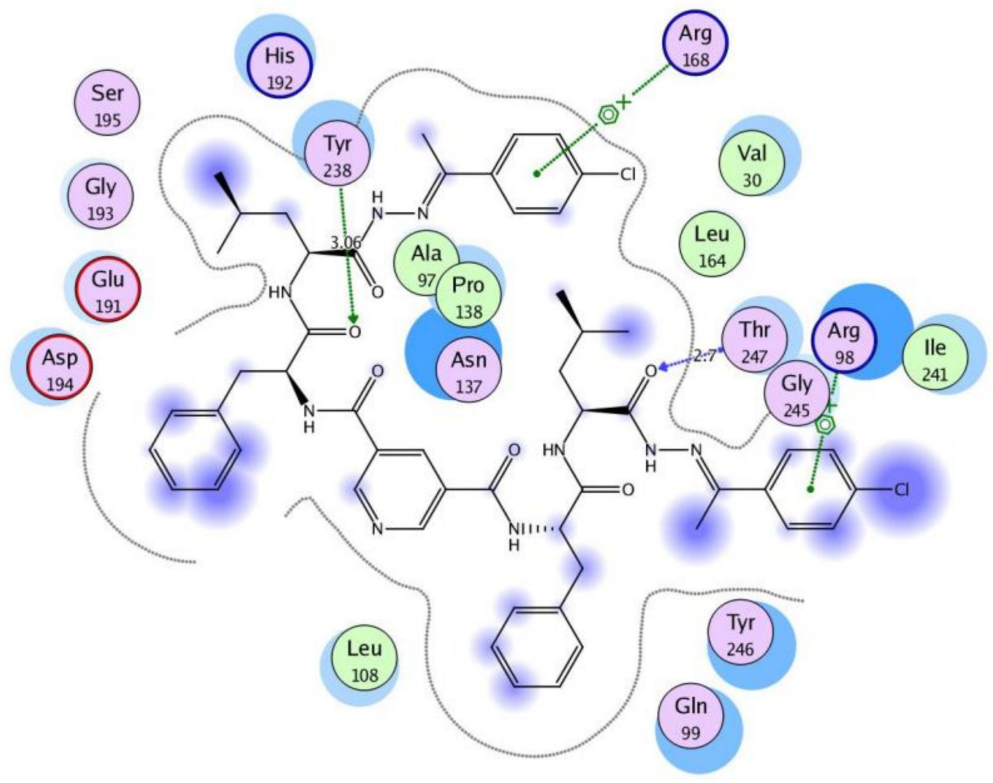

A

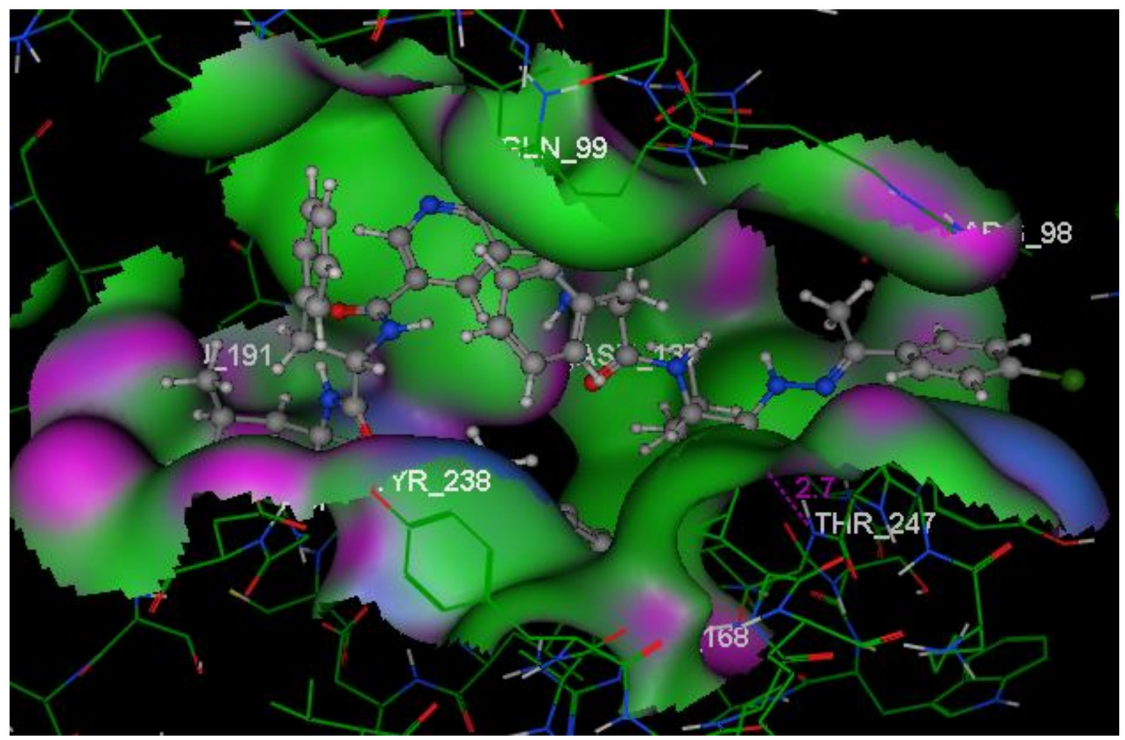

B

Figure 8. Two-dimensional (A) and 3D (B) patternsillustrating the proposed binding mode of the compound $5 \mathrm{c}$ in the LDHA binding pocket (PDB code: $4 \mathrm{ZVV})$. Hydrogen bonding interactions with the protein are shown as dashed lines. Green color reflects a hydrophobic area, pink color reflects a high polar area, and blue color reflects a mild polar area.

\section{Materials and Methods}

\subsection{Chemistry}

Melting points were determined in open glass capillary tubes with an electro thermal digital melting point apparatus (model: IA9100), and are uncorrected. Elemental microanalysis for carbon, hydrogen, and nitrogen (Microanalytical Unit, NRC) was found within the acceptable limits of the calculated values. Infrared spectra $(\mathrm{KBr})$ were recorded on a Nexus 670 FTIR Nicolet, Fourier Transform infrared spectrometer. Proton and carbon nuclear magnetic resonance $\left({ }^{1} \mathrm{H}-\right.$ and $\left.{ }^{13} \mathrm{C} N M R\right)$ spectra 
were run in (DMSO- $\mathrm{d}_{6}$ ) on Jeol $500 \mathrm{MHz}$ instruments. Mass spectra were run on a MAT Finnigan SSQ 7000 spectrometer, using the electron impact technique (EI). Analytical thin layer chromatography (TLC) was performed on silica gel aluminum sheets, $60 \mathrm{~F}_{254}$ (E. Merck). Compound 2 was prepared according to our previous reported procedure [39].

Synthesis of $\mathbf{N}^{\alpha}$-dinicotinoyl-bis[L-phenylalaninyl-L-leucyl]cycloalkanonehydrazones 3a-d. A mixture of hydrazide 2 ( $1 \mathrm{mmol})$ and cycloalkanone, namely, cyclopentanone, cyclohexanone, cycloheptanone, or cyclooctanone $(2 \mathrm{mmol})$, in glacial acetic acid $(30 \mathrm{~mL})$ was refluxed for $6 \mathrm{~h}$. The reaction mixture was poured onto ice water; the formed precipitate was filtered off, dried, and crystallized from $\mathrm{AcOH} / \mathrm{H}_{2} \mathrm{O}$ to give compounds $3 \mathbf{a}-\mathbf{d}$, respectively.

$\mathrm{N}^{3}, \mathrm{~N}^{5}$-Bis\{1-[(1-(2-cyclopentylidenehydrazinyl)-4-methyl-1-oxopentan-2-yl]amino)-1-oxo-3phenylpropan-2-yl\}pyridine-3,5-dicarboxamide (3a): Yield $72 \%, \operatorname{mp} 232-234{ }^{\circ} \mathrm{C}$. $[\alpha]_{\mathrm{D}}^{25}=-98(\mathrm{c}=$ 0.5, DMF). IR v, $\mathrm{cm}^{-1}:$ 3518-3334 (NH), 3090 (CH-Ar), 2985 (CH-aliph.), 1654, 1532, 1254 (C=O, amide I, II and III). ${ }^{1} \mathrm{H}$ NMR spectrum (DMSO-d $\left.\mathrm{d}_{6}\right), \delta_{\mathrm{H}}, \mathrm{ppm}: 0.90-0.95\left(\mathrm{~m}, 12 \mathrm{H}, 4 \mathrm{CH}_{3}\right), 1.24-1.35(\mathrm{~m}, 16 \mathrm{H}, 8$ $\left.\mathrm{CH}_{2}\right), 1.58-1.70\left(\mathrm{t}, 4 \mathrm{H}, 2 \mathrm{CH}_{2}\right), 2.18-2.25(\mathrm{~m}, 2 \mathrm{H}, 2 \mathrm{CH}), 3.42\left(\mathrm{~d}, 4 \mathrm{H}, 2 \mathrm{CH}_{2}\right), 4.35(\mathrm{t}, 2 \mathrm{H}, 2 \mathrm{CH}), 4.55(\mathrm{t}, 2 \mathrm{H}$, 2CH), 6.95-7.65 (m, 10H, 2Ph-H), 8.38, 9.05 (2s, 3H, pyr-H), 8.65, 8.75, 9.15 (3s, 6H, 6NH, exchangeable with $\left.\mathrm{D}_{2} \mathrm{O}\right) .{ }^{13} \mathrm{C}$ NMR spectrum $\left(\mathrm{DMSO}-\mathrm{d}_{6}\right), \delta_{\mathrm{C}}, \mathrm{ppm}: 17.32,17.54\left(4 \mathrm{C}, 4 \mathrm{CH}_{3}\right), 24.15,29.42,34.32$, 186.86 (10C, cyclopentyl ring), $23.98(2 \mathrm{C}, 2 \mathrm{CH}), 40.12\left(2 \mathrm{C}, 2 \mathrm{CH}_{2}\right), 42.26\left(2 \mathrm{C}, 2 \mathrm{CH}_{2}\right), 52.48,53.12(4 \mathrm{C}, 4$ $\mathrm{CH}), 124.18,128.26,129.35,138.64$ (12C, 2Ph-C), 131.55, 140.13, 152.12 (5C, pyr-C), 163.72, 169.15 (4C, 4CO-amide), 174.40 (2C, 2CO-hydrazone). MS (EI, $70 \mathrm{eV}): \mathrm{m} / \mathrm{z}(\%)=848(45)[\mathrm{M}]^{+}$. Found, \%: C 66.45; H 7.18; N 14.76. $\mathrm{C}_{47} \mathrm{H}_{61} \mathrm{~N}_{9} \mathrm{O}_{6}$ (848.04). Calculated, \%: C 66.57; H 7.25; N 14.86.

$\mathrm{N}^{3}, \mathrm{~N}^{5}$-Bis\{1-[(1-(2-cyclohexylidenehydrazinyl)-4-methyl-1-oxopentan-2-yl)amino]-1-oxo-3phenylpropan-2-yl\}pyridine-3,5-dicarboxamide (3b): Yield 65\%, mp 198-200 ${ }^{\circ} \mathrm{C}$. $[\alpha]_{\mathrm{D}}^{25}=-118(c=0.5$, DMF). IR v, cm ${ }^{-1}$ : 3510-3387 (NH), 3088 (CH-Ar), 2989 (CH-aliph.), 1655, 1533, 1254 (C=O, amide I, II and III). ${ }^{1} \mathrm{H}$ NMR spectrum (DMSO-d $\left.\mathrm{d}_{6}\right), \delta_{\mathrm{H}}, \mathrm{ppm}: 0.86-0.94\left(\mathrm{~m}, 12 \mathrm{H}, 4 \mathrm{CH}_{3}\right), 1.50-1.65\left(\mathrm{~m}, 16 \mathrm{H}, 8 \mathrm{CH}_{2}\right)$, 2.22-2.26 (m, $2 \mathrm{H}, 2 \mathrm{CH}), 2.10-2.14\left(\mathrm{~m}, 8 \mathrm{H}, 4 \mathrm{CH}_{2}\right), 3.46\left(\mathrm{~d}, 4 \mathrm{H}, 2 \mathrm{CH}_{2}\right), 4.34(\mathrm{t}, 2 \mathrm{H}, 2 \mathrm{CH}), 4.54(\mathrm{t}, 2 \mathrm{H}$, 2CH), 6.98-7.68 (m, 10H, 2Ph-H), 8.42, 9.09 (2s, 3H, pyr-H), 8.64, 8.78, 9.16 (3s, 6H, 6NH, exchangeable with $\left.\mathrm{D}_{2} \mathrm{O}\right) .{ }^{13} \mathrm{C}$ NMR spectrum (DMSO-d $\left.\mathrm{d}_{6}\right), \delta_{\mathrm{C}}, \mathrm{ppm}$ : $17.30,17.42\left(4 \mathrm{C}, 4 \mathrm{CH}_{3}\right), 24.76,27.02,28.32,165.85$ (12C, cyclohexyl ring), $23.90(2 \mathrm{C}, 2 \mathrm{CH}), 40.18\left(2 \mathrm{C}, 2 \mathrm{CH}_{2}\right), 42.34\left(2 \mathrm{C}, 2 \mathrm{CH}_{2}\right), 52.45,53.16(4 \mathrm{C}, 4 \mathrm{CH})$, 124.24, 128.28, 129.38, 138.65 (12C, 2Ph-C), 131.58, 140.15, 152.16 (5C, pyr-C), 163.76, 169.18, 174.42 (6C, 6 CO-amide). MS (EI, $70 \mathrm{eV}): \mathrm{m} / z(\%)=876(32)[\mathrm{M}]^{+}$. Found, \%: C 67.00; $\mathrm{H} 7.42 ; \mathrm{N} 14.30 . \mathrm{C}_{49} \mathrm{H}_{65} \mathrm{~N}_{9} \mathrm{O}_{6}$ (876.10). Calculated, \%: C 67.18; H 7.48; N 14.39.

$\mathrm{N}^{3}, \mathrm{~N}^{5}$-Bis\{1-[(1-(2-cycloheptylidenehydrazinyl)-4-methyl-1-oxopentan-2-yl)amino]-1-oxo-3phenylpropan-2-yl\}pyridine-3,5-dicarboxamide (3c): Yield 56\%, mp 221-223 ${ }^{\circ} \mathrm{C}$. $[\alpha]_{\mathrm{D}}^{25}=-96(c=$ 0.5, DMF). IR v, cm ${ }^{-1}:$ 3412-3375 (NH), 3092 (CH-Ar), 2995 (CH-aliph.), 1655, 1535, 1255 (C=O, amide I, II and III). ${ }^{1} \mathrm{H}$ NMR spectrum (DMSO-d $\left.\mathrm{d}_{6}\right), \delta_{\mathrm{H}}, \mathrm{ppm}:{ }^{1} \mathrm{H}$ NMR spectrum $\left(\right.$ DMSO-d $\left.\mathrm{d}_{6}\right), \delta_{\mathrm{H}}, \mathrm{ppm}$ : 0.88-0.96 (m, 12H, 4CH 3$), 1.50-1.70\left(\mathrm{~m}, 20 \mathrm{H}, 10 \mathrm{CH}_{2}\right), 2.20-2.24(\mathrm{~m}, 2 \mathrm{H}, 2 \mathrm{CH}), 2.02-2.10\left(\mathrm{~m}, 8 \mathrm{H}, 4 \mathrm{CH}_{2}\right)$, $3.45\left(\mathrm{~d}, 4 \mathrm{H}, 2 \mathrm{CH}_{2}\right), 4.44(\mathrm{t}, 2 \mathrm{H}, 2 \mathrm{CH}), 4.58(\mathrm{t}, 2 \mathrm{H}, 2 \mathrm{CH}), 6.92-7.60(\mathrm{~m}, 10 \mathrm{H}, 2 \mathrm{Ph}-\mathrm{H}), 8.54,9.00(2 \mathrm{~s}, 3 \mathrm{H}$, pyr-H), 8.65, 8.76, $9.15\left(3 \mathrm{~s}, 6 \mathrm{H}, 6 \mathrm{NH}\right.$, exchangeable with $\left.\mathrm{D}_{2} \mathrm{O}\right) .{ }^{13} \mathrm{C}$ NMR spectrum $\left(\mathrm{DMSO}-\mathrm{d}_{6}\right), \delta_{\mathrm{C}}$, ppm: 17.40, $17.44\left(4 \mathrm{C}, 4 \mathrm{CH}_{3}\right), 27.12,28.82,30.28,36.12,183.90$ (14C, cycloheptyl ring), $24.00(2 \mathrm{C}, 2 \mathrm{CH})$, $40.24\left(2 \mathrm{C}, 2 \mathrm{CH}_{2}\right), 42.46\left(2 \mathrm{C}, 2 \mathrm{CH}_{2}\right), 52.40,53.24(4 \mathrm{C}, 4 \mathrm{CH}), 124.28,128.30,129.42,138.64(12 \mathrm{C}, 2 \mathrm{Ph}-\mathrm{C})$, 131.60, 140.18, 152.18 (5C, pyr-C), 163.72, 169.24, 174.40 (6C, 6 CO-amide). MS (EI, $70 \mathrm{eV}): \mathrm{m} / z(\%)=902$ (14) $[\mathrm{M}-2]^{+}$. Found, \%: C 67.64; H 7.61; N 13.86. $\mathrm{C}_{51} \mathrm{H}_{69} \mathrm{~N}_{9} \mathrm{O}_{6}$ (904.15). Calculated, \%: C 67.75; H 7.69; N 13.94 .

$\mathrm{N}^{3}, \mathrm{~N}^{5}$-Bis $\{1-[(1-(2-c y c l o o c t y l i d e n e h y d r a z i n y l)-4-m e t h y l-1-o x o p e n t a n-2-y 1)$ amino]-1-oxo-3phenylpropan-2-yl\}pyridine-3,5-dicarboxamide (3d): Yield 56\%, mp $242-244{ }^{\circ} \mathrm{C}$. $[\alpha]_{\mathrm{D}}^{25}=-104(\mathrm{c}$ $=0.5, \mathrm{DMF}) . \mathrm{IR} v, \mathrm{~cm}^{-1}: 3418-3360(\mathrm{NH}), 3086$ (CH-Ar), 2990 (CH-aliph.), 1654, 1533, 1255 (C=O, amide I, II and III). ${ }^{1} \mathrm{H}$ NMR spectrum (DMSO- $\left.\mathrm{d}_{6}\right), \delta_{\mathrm{H}}, \mathrm{ppm}: 0.84-0.90\left(\mathrm{~m}, 12 \mathrm{H}, 4 \mathrm{CH}_{3}\right), 1.22-1.60(\mathrm{~m}$, $\left.20 \mathrm{H}, 10 \mathrm{CH}_{2}\right), 1.65-1.70\left(\mathrm{~m}, 4 \mathrm{H}, 2 \mathrm{CH}_{2}\right), 2.00-2.08\left(\mathrm{~m}, 8 \mathrm{H}, 4 \mathrm{CH}_{2}\right), 2.18-2.22(\mathrm{~m}, 2 \mathrm{H}, 2 \mathrm{CH}), 3.52(\mathrm{~d}, 4 \mathrm{H}$, 
$\left.2 \mathrm{CH}_{2}\right), 4.20-4.35(\mathrm{~m}, 2 \mathrm{H}, 2 \mathrm{CH}), 4.60-4.68(\mathrm{~m}, 2 \mathrm{H}, 2 \mathrm{CH}), 6.96-7.54(\mathrm{~m}, 10 \mathrm{H}, 2 \mathrm{Ph}-\mathrm{H}), 8.46,9.05(2 \mathrm{~s}, 3 \mathrm{H}$, pyr-H), 8.68, 8.74, $9.12\left(3 \mathrm{~s}, 6 \mathrm{H}, 6 \mathrm{NH}\right.$, exchangeable with $\left.\mathrm{D}_{2} \mathrm{O}\right) .{ }^{13} \mathrm{C}$ NMR spectrum (DMSO- $\left.\mathrm{d}_{6}\right), \delta_{\mathrm{C}}$, ppm: 17.30, $17.54\left(4 \mathrm{C}, 4 \mathrm{CH}_{3}\right), 22.92,24.80,25.28,27.02,28.79,154.94$ (16C, cyclooctyl ring), 23.68 (2C, $2 \mathrm{CH}), 40.10\left(2 \mathrm{C}, 2 \mathrm{CH}_{2}\right), 42.56\left(2 \mathrm{C}, \mathrm{CH}_{2}\right), 52.62,53.12(4 \mathrm{C}, 4 \mathrm{CH}), 124.24,128.25,129.40,138.65(12 \mathrm{C}$, 2Ph-C), 131.70, 140.34, 152.25 (5C, pyr-C), 163.68, 169.16, 174.45 (6C, 6 CO-amide). MS (EI, $70 \mathrm{eV}): \mathrm{m} / \mathrm{z}$ $(\%)=932(32)[\mathrm{M}]^{+}$. Found, \%: C 68.18; H 7.80; N 13.45. $\mathrm{C}_{53} \mathrm{H}_{73} \mathrm{~N}_{9} \mathrm{O}_{6}$ (932.22). Calculated, \%: C 68.29; H 7.89; N 13.52 .

Synthesis of $\mathrm{N}^{\alpha}$-dinicotinoyl-bis[L-phenylalaninyl-L-leucyl] Schiff bases $4 \mathrm{a}-\mathrm{c}$, $5 \mathrm{a}-\mathrm{e}$, and $\mathbf{6 a}, \mathrm{b}$. A mixture of $2(1 \mathrm{mmol})$ and acetylpyridines or substituted acetophenones or heterocyclic aldehyde, namely, 2-, 3-, 4-acetylpyridine,4-methyl-, 4-methoxy-, 4-chloro-, 4-bromo-, 4-nitroacetophenone or 2-furane, 2-thiophene aldeydes $(2 \mathrm{mmol})$ in $\mathrm{AcOH}$ or ethanol $(30 \mathrm{~mL})$ was refluxed for $4-6 \mathrm{~h}$, and then evaporated to dryness. The formed residue was solidified with $n$-hexane, filtered off, and crystallized from the proper solvents to give Schiff base derivatives $\mathbf{4 a - c}, \mathbf{5 a}-\mathbf{e}, \mathbf{a n d} \mathbf{6} \mathbf{a}, \mathbf{b}$, respectively.

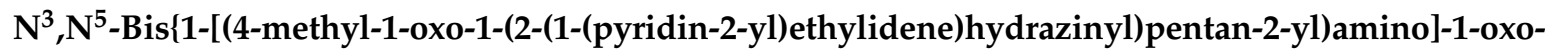
3-phenylpropan-2-yl\}pyridine-3,5-dicarboxamide (4a): Yield 60\%, mp 216-218 ${ }^{\circ} \mathrm{C}\left(\mathrm{DMF} / \mathrm{H}_{2} \mathrm{O}\right) .[\alpha]_{\mathrm{D}}^{25}$ $=-112$ ( $c=0.5$, DMF). IR $v, \mathrm{~cm}^{-1}:$ 3450-3354 (NH), 3092 (CH-Ar), 2986 (CH-aliph.), 1654, 1534, 1252 (C=O, amide I, II and III). ${ }^{1} \mathrm{H}$ NMR spectrum $\left(\mathrm{DMSO}_{-} \mathrm{d}_{6}\right), \delta_{\mathrm{H}}, \mathrm{ppm}: 0.85-0.95\left(\mathrm{~m}, 12 \mathrm{H}, 4 \mathrm{CH}_{3}\right), 1.60-1.70$ $\left(\mathrm{m}, 4 \mathrm{H}, 2 \mathrm{CH}_{2}\right), 1.82\left(\mathrm{~s}, 6 \mathrm{H}, 2 \mathrm{CH}_{3}\right), 2.20-2.30(\mathrm{~m}, 2 \mathrm{H}, 2 \mathrm{CH}), 3.48\left(\mathrm{~d}, 4 \mathrm{H}, 2 \mathrm{CH}_{2}\right), 4.25-4.34(\mathrm{~m}, 2 \mathrm{H}, 2 \mathrm{CH})$, 4.60-4.70 (m, 2H, 2CH), 6.92-7.65 (m, 10H, 2Ph-H), 7.68-8.50 (m, 10H, pyr-H), 9.04 (s, 1H, pyr-H-4), $8.65,8.85,9.15\left(3 \mathrm{~s}, 6 \mathrm{H}, 6 \mathrm{NH}\right.$, exchangeable with $\left.\mathrm{D}_{2} \mathrm{O}\right) .{ }^{13} \mathrm{C}$ NMR spectrum $\left(\mathrm{DMSO}-\mathrm{d}_{6}\right), \delta_{\mathrm{C}}$, ppm: 13.45 , $17.85,18.00\left(6 \mathrm{C}, 6 \mathrm{CH}_{3}\right), 23.96(2 \mathrm{C}, 2 \mathrm{CH}), 40.56\left(2 \mathrm{C}, 2 \mathrm{CH}_{2}\right), 42.44\left(2 \mathrm{C}, \mathrm{CH}_{2}\right), 52.56,53.12(4 \mathrm{C}, 4 \mathrm{CH})$, $124.24,128.26,129.35,138.68(12 \mathrm{C}, 2 \mathrm{Ph}-\mathrm{C}), 145.30(2 \mathrm{C}, 2 \mathrm{C}=\mathrm{N}), 122.90,126.00,131.60,135.80,140.20$, 148.62, 152.22, 154.48 (15C, 3 pyr-C), 163.82, 169.33 (4C, 4 CO-amide), 174.55 (2C, 2CO-hydrazone). MS (EI, $70 \mathrm{eV}): m / z(\%)=922(54)[\mathrm{M}]^{+}$. Found, \%: C 66.32; H 6.36; N 16.60. $\mathrm{C}_{51} \mathrm{H}_{59} \mathrm{~N}_{11} \mathrm{O}_{6}(922.08)$. Calculated, \%: C 66.43; H 6.45; N 16.71.

$\mathrm{N}^{3}, \mathrm{~N}^{5}$-Bis\{1-[(4-methyl-1-oxo-1-(2-(1-(pyridin-3-yl)ethylidene)hydrazinyl)pentan-2-yl)-amino]-1-oxo3-phenylpropan-2-yl\}pyridine-3,5-dicarboxamide (4b): Yield $58 \%$, mp $208-210{ }^{\circ} \mathrm{C}\left(\mathrm{AcOH} / \mathrm{H}_{2} \mathrm{O}\right) .[\alpha]_{\mathrm{D}}^{25}$ $=-132(c=0.5, \mathrm{DMF})$. IR $v, \mathrm{~cm}^{-1}:$ 3467-3350 (NH), 3075 (CH-Ar), 2990 (CH-aliph.), 1652, 1536, 1252 (C=O, amide I, II and III). ${ }^{1} \mathrm{H}$ NMR spectrum $\left(\mathrm{DMSO}^{\mathrm{d}} \mathrm{d}_{6}\right), \delta_{\mathrm{H}}, \mathrm{ppm}: 0.88-0.93\left(\mathrm{~m}, 12 \mathrm{H}, 4 \mathrm{CH}_{3}\right), 1.62-1.74$ $\left(\mathrm{m}, 4 \mathrm{H}, 2 \mathrm{CH}_{2}\right), 1.80\left(\mathrm{~s}, 6 \mathrm{H}, 2 \mathrm{CH}_{3}\right), 2.16-2.24(\mathrm{~m}, 2 \mathrm{H}, 2 \mathrm{CH}), 3.45\left(\mathrm{~d}, 4 \mathrm{H}, 2 \mathrm{CH}_{2}\right), 4.24-4.35(\mathrm{~m}, 2 \mathrm{H}, 2 \mathrm{CH})$, 4.62-4.70 (m, 2H, 2CH), 6.96-7.60 (m, 10H, 2Ph-H), 7.72-8.65 (m, 8H, pyr-H), 9.04, 9.08 (3s, 3H, pyr-H), 8.70, 8.86, $9.18\left(3 \mathrm{~s}, 6 \mathrm{H}, 6 \mathrm{NH}\right.$, exchangeable with $\left.\mathrm{D}_{2} \mathrm{O}\right) .{ }^{13} \mathrm{C}$ NMR spectrum (DMSO-d $\left.\mathrm{d}_{6}\right), \delta_{\mathrm{C}}, \mathrm{ppm}: 15.25$, $17.88,18.02\left(6 \mathrm{C}, 6 \mathrm{CH}_{3}\right), 23.94(2 \mathrm{C}, 2 \mathrm{CH}), 40.55\left(2 \mathrm{C}, 2 \mathrm{CH}_{2}\right), 42.45\left(2 \mathrm{C}, \mathrm{CH}_{2}\right), 52.55,53.15(4 \mathrm{C}, 4 \mathrm{CH})$, $124.25,128.25,129.35,138.65$ (12C, 2Ph-C), $145.35(2 \mathrm{C}, 2 \mathrm{C}=\mathrm{N}), 122.96,126.14,131.65,137.56,140.45$, 147.82, 151.32, 154.80 (15C, 3 pyr-C), 163.80, 169.14 (4C, 4CO-amide), 168.12 (2C, 2CO-hydrazone). MS (EI, $70 \mathrm{eV}): \mathrm{m} / \mathrm{z}(\%)=921(18)[\mathrm{M}-1]^{+}$. Found, \%: C 66.33; H 6.34; $\mathrm{N} 16.64 . \mathrm{C}_{51} \mathrm{H}_{59} \mathrm{~N}_{11} \mathrm{O}_{6}(922.08)$. Calculated, \%: C 66.43; H 6.45; N 16.71.

$\mathrm{N}^{3}, \mathrm{~N}^{5}$-Bis\{1-[(4-methyl-1-oxo-1-(2-(1-(pyridin-4-yl)ethylidene)hydrazinyl)pentan-2-yl)-amino]-1-oxo3-phenylpropan-2-yl\}pyridine-3,5-dicarboxamide (4c): Yield 75\%, mp $196-198{ }^{\circ} \mathrm{C}\left(\mathrm{EtOH} / \mathrm{H}_{2} \mathrm{O}\right) .[\alpha]_{\mathrm{D}}^{25}=$ -140 ( $c=0.5, \mathrm{DMF})$. IR $v, \mathrm{~cm}^{-1}:$ 3466-3348 (NH), 3080 (CH-Ar), 2986 (CH-aliph.), 1655, 1535, 1255 $\left(\mathrm{C}=\mathrm{O}\right.$, amide I, II and III). ${ }^{1} \mathrm{H}$ NMR spectrum $\left(\mathrm{DMSO}_{\mathrm{d}}\right), \mathrm{d}_{\mathrm{H}}, \mathrm{ppm}: 0.86-0.90\left(\mathrm{~m}, 12 \mathrm{H}, 4 \mathrm{CH}_{3}\right), 1.65-1.70$ $\left(\mathrm{m}, 4 \mathrm{H}, 2 \mathrm{CH}_{2}\right), 1.96\left(\mathrm{~s}, 6 \mathrm{H}, 2 \mathrm{CH}_{3}\right), 2.18-2.20(\mathrm{~m}, 2 \mathrm{H}, 2 \mathrm{CH}), 3.46\left(\mathrm{~d}, 4 \mathrm{H}, 2 \mathrm{CH}_{2}\right), 4.25-4.32(\mathrm{~m}, 2 \mathrm{H}, 2 \mathrm{CH})$, 4.60-4.68 (m, 2H, 2CH), 6.98-7.64 (m, 10H, 2Ph-H), 7.70-8.65 (m, 10H, pyr-H), 9.06 (s, 1H, pyr-H), 8.75, 8.85, $9.14\left(3 \mathrm{~s}, 6 \mathrm{H}, 6 \mathrm{NH}\right.$, exchangeable with $\left.\mathrm{D}_{2} \mathrm{O}\right) .{ }^{13} \mathrm{C}$ NMR spectrum $\left(\mathrm{DMSO}-\mathrm{d}_{6}\right), \delta_{\mathrm{C}}, \mathrm{ppm}: 16.02$, $17.86,18.12\left(6 \mathrm{C}, 6 \mathrm{CH}_{3}\right), 23.86(2 \mathrm{C}, 2 \mathrm{CH}), 40.50\left(2 \mathrm{C}, 2 \mathrm{CH}_{2}\right), 42.60\left(2 \mathrm{C}, \mathrm{CH}_{2}\right), 52.54,53.18(4 \mathrm{C}, 4 \mathrm{CH})$, 124.60, 128.42, 129.38, 138.658 (12C, 2Ph-C), 146.30 (2C, 2C=N), 124.14, 131.60, 138.50, 140.40, 147.50, 149.54 (15C, 3 pyr-C), 164.15, 169.46 (4C, 4CO-amide), 174.10 (2C, 2CO-hydrazone). MS (EI, $70 \mathrm{eV}): \mathrm{m} / \mathrm{z}$ 
$(\%)=920(8)[\mathrm{M}-2]^{+}$. Found, \%: C 66.37; H 6.35; N 16.65. $\mathrm{C}_{51} \mathrm{H}_{59} \mathrm{~N}_{11} \mathrm{O}_{6}$ (922.08). Calculated, \%: C $66.43 ; \mathrm{H} 6.45 ; \mathrm{N} 16.71$.

$\mathrm{N}^{3}, \mathrm{~N}^{5}$-Bis\{1-[(1-(2-(1-(4-methoxyphenyl)ethylidene)hydrazinyl)-4-methyl-1-oxopentan-2-yl)-amino]1-oxo-3-phenylpropan-2-yl\}pyridine-3,5-dicarboxamide (5a): Yield 55\%, mp 202-204 ${ }^{\circ} \mathrm{C}\left(\mathrm{AcOH} / \mathrm{H}_{2} \mathrm{O}\right)$. $[\alpha]_{\mathrm{D}}^{25}=-110(c=0.5, \mathrm{DMF}) . \mathrm{IR} v, \mathrm{~cm}^{-1}: 3478-3348(\mathrm{NH}), 3085$ (CH-Ar), 2976 (CH-aliph.), 1655, 1534, $1253\left(\mathrm{C}=\mathrm{O}\right.$, amide I, II and III). ${ }^{1} \mathrm{H}$ NMR spectrum $\left(\mathrm{DMSO}_{-} \mathrm{d}_{6}\right), \delta_{\mathrm{H}}, \mathrm{ppm}: 0.86-0.94\left(\mathrm{~m}, 12 \mathrm{H}, 4 \mathrm{CH}_{3}\right)$, $1.62-1.74\left(\mathrm{~m}, 4 \mathrm{H}, 2 \mathrm{CH}_{2}\right), 1.98\left(\mathrm{~s}, 6 \mathrm{H}, 2 \mathrm{CH}_{3}\right), 2.18-2.25(\mathrm{~m}, 2 \mathrm{H}, 2 \mathrm{CH}), 3.45\left(\mathrm{~d}, 4 \mathrm{H}, 2 \mathrm{CH}_{2}\right), 3.68(\mathrm{~s}, 6 \mathrm{H}$, $\left.2 \mathrm{OCH}_{3}\right), 4.20-4.35(\mathrm{~m}, 2 \mathrm{H}, 2 \mathrm{CH}), 4.65-4.70(\mathrm{~m}, 2 \mathrm{H}, 2 \mathrm{CH}), 6.95-7.65(\mathrm{~m}, 18 \mathrm{H}, 4 \mathrm{Ph}-\mathrm{H}), 8.62,9.00(2 \mathrm{~s}$, $3 \mathrm{H}$, pyr-H) $, 8.66,8.76,9.20\left(3 \mathrm{~s}, 6 \mathrm{H}, 6 \mathrm{NH}\right.$, exchangeable with $\left.\mathrm{D}_{2} \mathrm{O}\right) .{ }^{13} \mathrm{C}$ NMR spectrum (DMSO-d 6 ), $\delta_{\mathrm{C}}$, ppm: 15.50, 17.85, $18.24\left(6 \mathrm{C}, 6 \mathrm{CH}_{3}\right), 23.78(2 \mathrm{C}, 2 \mathrm{CH}), 40.26\left(2 \mathrm{C}, 2 \mathrm{CH}_{2}\right), 42.48\left(2 \mathrm{C}, \mathrm{CH}_{2}\right), 52.76$, $53.15(4 \mathrm{C}, 4 \mathrm{CH}), 55.72\left(2 \mathrm{C}, 2 \mathrm{OCH}_{3}\right), 114.32,125.65,127.28,128.32,128.65,129.75,138.68,162.84(24 \mathrm{C}$, $4 \mathrm{Ph}-\mathrm{C}), 131.60,140.12,152.20$ (5C, pyr-C), 145.15 (2C, 2C=N), 164.14, 169.65 (4C, 4 CO-amide), 174.06 (2C, 2CO-hydrazone). MS (EI, $70 \mathrm{eV}): \mathrm{m} / z(\%)=980(16)[\mathrm{M}]^{+}$. Found, \%: C 67.28; H 6.62; N 12.76 . $\mathrm{C}_{55} \mathrm{H}_{65} \mathrm{~N}_{9} \mathrm{O}_{8}$ (980.16). Calculated, \%: C 67.40; $\mathrm{H} 6.68 ; \mathrm{N} 12.86$.

$\mathrm{N}^{3}, \mathrm{~N}^{5}$-Bis\{1-[(1-(2-(1-(4-chlorophenyl)ethylidene)hydrazinyl)-4-methyl-1-oxopentan-2-yl)-amino]1-oxo-3-phenylpropan-2-yl\}pyridine-3,5-dicarboxamide (5b): Yield $60 \%$, mp 184-186 ${ }^{\circ} \mathrm{C}\left(\mathrm{DMF} / \mathrm{H}_{2} \mathrm{O}\right)$. $[\alpha]_{\mathrm{D}}^{25}=-99(c=0.5, \mathrm{DMF})$. IR $v, \mathrm{~cm}^{-1}:$ 3467-3345 (NH), 3086 (CH-Ar), 2979 (CH-aliph.), 1654, 1535, $1254\left(\mathrm{C}=\mathrm{O}\right.$, amide I, II and III). ${ }^{1} \mathrm{H}$ NMR spectrum $\left(\mathrm{DMSO}_{6} \mathrm{~d}_{6}\right), \delta_{\mathrm{H}}, \mathrm{ppm}: 0.88-0.92\left(\mathrm{~m}, 12 \mathrm{H}, 4 \mathrm{CH}_{3}\right)$, $1.64-1.70\left(\mathrm{~m}, 4 \mathrm{H}, 2 \mathrm{CH}_{2}\right), 2.05\left(\mathrm{~s}, 6 \mathrm{H}, 2 \mathrm{CH}_{3}\right), 2.20-2.24(\mathrm{~m}, 2 \mathrm{H}, 2 \mathrm{CH}), 3.46\left(\mathrm{~d}, 4 \mathrm{H}, 2 \mathrm{CH}_{2}\right), 4.26-4.34(\mathrm{~m}$, $2 \mathrm{H}, 2 \mathrm{CH}), 4.68-4.72(\mathrm{~m}, 2 \mathrm{H}, 2 \mathrm{CH}), 7.10-7.90(\mathrm{~m}, 18 \mathrm{H}, 4 \mathrm{Ph}-\mathrm{H}), 8.65,9.04(2 \mathrm{~s}, 3 \mathrm{H}, \mathrm{pyr}-\mathrm{H}), 8.70,8.78,9.18$ $\left(3 \mathrm{~s}, 6 \mathrm{H}, 6 \mathrm{NH}\right.$, exchangeable with $\left.\mathrm{D}_{2} \mathrm{O}\right) .{ }^{13} \mathrm{C}$ NMR spectrum $\left(\mathrm{DMSO}-\mathrm{d}_{6}\right), \delta_{\mathrm{C}}, \mathrm{ppm}: 15.85,17.80,18.12$ $\left(6 \mathrm{C}, 6 \mathrm{CH}_{3}\right), 23.84(2 \mathrm{C}, 2 \mathrm{CH}), 40.38\left(2 \mathrm{C}, 2 \mathrm{CH}_{2}\right), 42.50\left(2 \mathrm{C}, \mathrm{CH}_{2}\right), 52.74,53.10(4 \mathrm{C}, 4 \mathrm{CH}), 125.65,127.28$, $128.32,128.78,129.05,134.95,135.68,136.84$ (24C, 4Ph-C), 131.70, 140.18, 152.26 (5C, pyr-C), 144.98 (2C, $2 \mathrm{C}=\mathrm{N}), 164.34,169.65$ (4C, 4 CO-amide), 173.96 (2C, 2CO-hydrazone). MS (EI, $70 \mathrm{eV}): \mathrm{m} / \mathrm{z}(\%)=988$ (24) $[\mathrm{M}-1]^{+}$. Found, \%: C 64.24; $\mathrm{H} 5.90 ; \mathrm{Cl} 7.08 ; \mathrm{N} 12.65 . \mathrm{C}_{53} \mathrm{H}_{59} \mathrm{Cl}_{2} \mathrm{~N}_{9} \mathrm{O}_{6}$ (988.99). Calculated, \%: C 64.36; H 6.01; Cl 7.17; N 12.75.

$\mathrm{N}^{3}, \mathrm{~N}^{5}$-Bis\{1-[(1-(2-(1-(4-bromophenyl)ethylidene)hydrazinyl)-4-methyl-1-oxopentan-2-yl)-amino]1-oxo-3-phenylpropan-2-yl\}pyridine-3,5-dicarboxamide (5c): Yield $72 \%$, mp 210-212 ${ }^{\circ} \mathrm{C}\left(\mathrm{DMF} / \mathrm{H}_{2} \mathrm{O}\right)$. $[\alpha]_{\mathrm{D}}^{25}=-124(c=0.5, \mathrm{DMF}) . \mathrm{IR} v, \mathrm{~cm}^{-1}:$ 3462-3345 (NH), 3090 (CH-Ar), 2988 (CH-aliph.), 1655, 1532, $1250\left(\mathrm{C}=\mathrm{O}\right.$, amide I, II and III). ${ }^{1} \mathrm{H}$ NMR spectrum (DMSO-d $\left.\mathrm{d}_{6}\right), \delta_{\mathrm{H}}, \mathrm{ppm}: 0.84-0.90\left(\mathrm{~m}, 12 \mathrm{H}, 4 \mathrm{CH}_{3}\right)$, 1.60-1.65 (m, $\left.4 \mathrm{H}, 2 \mathrm{CH}_{2}\right), 2.02\left(\mathrm{~s}, 6 \mathrm{H}, 2 \mathrm{CH}_{3}\right), 2.18-2.22(\mathrm{~m}, 2 \mathrm{H}, 2 \mathrm{CH}), 3.48\left(\mathrm{~d}, 4 \mathrm{H}, 2 \mathrm{CH}_{2}\right), 4.25-4.35(\mathrm{~m}$, $2 \mathrm{H}, 2 \mathrm{CH}), 4.66-4.70(\mathrm{~m}, 2 \mathrm{H}, 2 \mathrm{CH}), 7.14-7.82(\mathrm{~m}, 18 \mathrm{H}, 4 \mathrm{Ph}-\mathrm{H}), 8.62,9.08$ (2s, 3H, pyr-H), 8.72, 8.76, 9.16 $\left(3 \mathrm{~s}, 6 \mathrm{H}, 6 \mathrm{NH}\right.$, exchangeable with $\left.\mathrm{D}_{2} \mathrm{O}\right) .{ }^{13} \mathrm{C}$ NMR spectrum $\left(\mathrm{DMSO}-\mathrm{d}_{6}\right), \delta_{\mathrm{C}}, \mathrm{ppm}: 15.90,17.60,17.86$ $\left(6 \mathrm{C}, 6 \mathrm{CH}_{3}\right), 23.80(2 \mathrm{C}, 2 \mathrm{CH}), 40.42\left(2 \mathrm{C}, 2 \mathrm{CH}_{2}\right), 42.56\left(2 \mathrm{C}, \mathrm{CH}_{2}\right), 52.78,53.15(4 \mathrm{C}, 4 \mathrm{CH}), 125.55,126.04$, 127.56, 128.24, 128.70, 130.75, 135.98, 136.34 (24C, 4Ph-C), 131.62, 140.26, 152.28 (5C, pyr-C), 145.08 (2C, $2 \mathrm{C}=\mathrm{N}), 164.65,168.98$ (4C, 4 CO-amide), 174.16 (2C, 2CO-hydrazone). MS (EI, $70 \mathrm{eV}): \mathrm{m} / \mathrm{z}(\%)=1078$ (14) $[\mathrm{M}]^{+}$. Found, \%: C 58.88; H 5.45; N 11.62. $\mathrm{C}_{53} \mathrm{H}_{59} \mathrm{Br}_{2} \mathrm{~N}_{9} \mathrm{O}_{6}$ (1077.90). Calculated, \%: C 59.06; H $5.52 ; \mathrm{N} 11.69$.

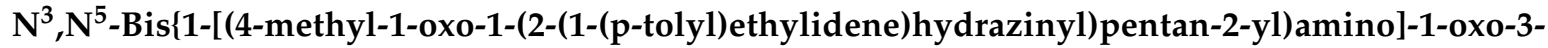
phenylpropan-2-yl\}pyridine-3,5-dicarboxamide (5d): Yield 55\%, mp $235-237{ }^{\circ} \mathrm{C}\left(\mathrm{DMF} / \mathrm{H}_{2} \mathrm{O}\right) .[\alpha]_{\mathrm{D}}^{25}$ $=-78$ ( $c=0.5, \mathrm{DMF}$ ). IR $v, \mathrm{~cm}^{-1}:$ 3465-3340 (NH), 3088 (CH-Ar), 2980 (CH-aliph.), 1656, 1536, 1256 (C=O, amide I, II, and III). ${ }^{1} \mathrm{H}$ NMR spectrum $\left(\right.$ DMSO- $\left._{6}\right), \delta_{\mathrm{H}}, \mathrm{ppm}: 0.88-0.92\left(\mathrm{~m}, 12 \mathrm{H}, 4 \mathrm{CH}_{3}\right), 1.66-1.70$ $\left(\mathrm{m}, 4 \mathrm{H}, 2 \mathrm{CH}_{2}\right), 2.00\left(\mathrm{~s}, 6 \mathrm{H}, 2 \mathrm{CH}_{3}\right), 2.18-2.24(\mathrm{~m}, 2 \mathrm{H}, 2 \mathrm{CH}), 2.34\left(\mathrm{~s}, 6 \mathrm{H}, 2 \mathrm{CH}_{3}\right), 3.43\left(\mathrm{~d}, 4 \mathrm{H}, 2 \mathrm{CH}_{2}\right)$, $4.23-4.30(\mathrm{~m}, 2 \mathrm{H}, 2 \mathrm{CH}), 4.66-4.72(\mathrm{~m}, 2 \mathrm{H}, 2 \mathrm{CH}), 7.12-7.68(\mathrm{~m}, 18 \mathrm{H}, 4 \mathrm{Ph}-\mathrm{H}), 8.65,9.05(2 \mathrm{~s}, 3 \mathrm{H}, \mathrm{pyr}-\mathrm{H})$, 8.68, 8.75, $9.18\left(3 \mathrm{~s}, 6 \mathrm{H}, 6 \mathrm{NH}\right.$, exchangeable with $\left.\mathrm{D}_{2} \mathrm{O}\right) .{ }^{13} \mathrm{C}$ NMR spectrum (DMSO-d $\left.{ }_{6}\right), \delta_{\mathrm{C}}$, ppm: 16.80 , 17.90, 18.15, $21.12\left(8 \mathrm{C}, 8 \mathrm{CH}_{3}\right), 23.86(2 \mathrm{C}, 2 \mathrm{CH}), 40.35\left(2 \mathrm{C}, 2 \mathrm{CH}_{2}\right), 42.52\left(2 \mathrm{C}, \mathrm{CH}_{2}\right), 52.75,53.18(4 \mathrm{C}, 4$ $\mathrm{CH}), 125.72,126.45,127.35,128.40,128.92,134.08,136.10,139.56$ (24C, 4Ph-C), 131.68, 140.25, 152.25 (5C, pyr-C), $145.60(2 \mathrm{C}, 2 \mathrm{C}=\mathrm{N}), 164.12,169.68$ (4C, $4 \mathrm{CO}$-amide), 174.18 (2C, 2CO-hydrazone). MS (EI, 70 
$\mathrm{eV}): m / z(\%)=948(42)[\mathrm{M}]^{+}$. Found, \%: $\mathrm{C}, 69.60 ; \mathrm{H}, 6.86 ; \mathrm{N}, 13.22 . \mathrm{C}_{55} \mathrm{H}_{65} \mathrm{~N}_{9} \mathrm{O}_{6}$ (948.18). Calculated, $\%:$ C, 69.67; H, 6.91; N, 13.30 .

$\mathbf{N}^{3}, \mathbf{N}^{5}$-Bis\{1-[(4-methyl-1-(2-(1-(4-nitrophenyl)ethylidene)hydrazinyl)-1-oxopentan-2-yl)-amino]-1-oxo3-phenylpropan-2-yl\}pyridine-3,5-dicarboxamide (5e). Yield $72 \%, \mathrm{mp} 256-258{ }^{\circ} \mathrm{C}\left(\mathrm{DMF} / \mathrm{H}_{2} \mathrm{O}\right) .[\alpha]_{\mathrm{D}}^{25}$ $=-104(c=0.5, \mathrm{DMF}) . \mathrm{IR} v, \mathrm{~cm}^{-1}:$ 3485-3354 (NH), $3092(\mathrm{CH}-\mathrm{Ar}), 2986(\mathrm{CH}-$ aliph.), 1655, 1533, $1255\left(\mathrm{C}=\mathrm{O}\right.$, amide I, II, and III). ${ }^{1} \mathrm{H}$ NMR spectrum $\left(\mathrm{DMSO}^{\mathrm{d}} \mathrm{d}_{6}\right), \delta_{\mathrm{H}}, \mathrm{ppm}: 0.86-0.90\left(\mathrm{~m}, 12 \mathrm{H}, 4 \mathrm{CH}_{3}\right)$, 1.66-1.74 (m, $\left.4 \mathrm{H}, 2 \mathrm{CH}_{2}\right), 2.10\left(\mathrm{~s}, 6 \mathrm{H}, 2 \mathrm{CH}_{3}\right), 2.24-2.26(\mathrm{~m}, 2 \mathrm{H}, 2 \mathrm{CH}), 3.45\left(\mathrm{~d}, 4 \mathrm{H}, 2 \mathrm{CH}_{2}\right), 4.25-4.32(\mathrm{~m}$, $2 \mathrm{H}, 2 \mathrm{CH}), 4.65-4.68(\mathrm{~m}, 2 \mathrm{H}, 2 \mathrm{CH}), 7.08-7.26(\mathrm{~m}, 10 \mathrm{H}, 2 \mathrm{Ph}-\mathrm{H}), 8.00-8.28(\mathrm{~m}, 8 \mathrm{H}, 2 \mathrm{Ph}-\mathrm{H}), 8.58,9.03$ (2s, $3 \mathrm{H}$, pyr-H), 8.65, 8.75, $9.15\left(3 \mathrm{~s}, 6 \mathrm{H}, 6 \mathrm{NH}\right.$, exchangeable with $\left.\mathrm{D}_{2} \mathrm{O}\right) .{ }^{13} \mathrm{C}$ NMR spectrum $\left(\right.$ DMSO-d $\left.\mathrm{d}_{6}\right), \delta_{\mathrm{C}}$, ppm: 16.04, 17.88, $18.06\left(6 \mathrm{C}, 6 \mathrm{CH}_{3}\right), 23.95(2 \mathrm{C}, 2 \mathrm{CH}), 40.45\left(2 \mathrm{C}, 2 \mathrm{CH}_{2}\right), 42.54\left(2 \mathrm{C}, \mathrm{CH}_{2}\right), 52.73,53.13$ (4C, $4 \mathrm{CH}), 125.63,126.86,127.32,127.90,128.24,136.70,143.06,149.85$ (24C, 4Ph-C), 131.55, 140.25, 152.20 (5C, pyr-C), 145.00 (2C, 2C=N), 163.98, 169.40 (4C, 4 CO-amide), 174.34 (2C, 2CO-hydrazone). MS (EI, $70 \mathrm{eV}): \mathrm{m} / \mathrm{z}(\%)=1010(8)[\mathrm{M}-1]^{+}$. Found, \%: C, 62.94; H, 5.80; N, 15.20. $\mathrm{C}_{53} \mathrm{H}_{59} \mathrm{~N}_{11} \mathrm{O}_{10}(1010.12)$. Calculated, \%: C, 63.02; H, 5.89; N, 15.25.

$\mathrm{N}^{3}$, $\mathrm{N}^{5}$-bis $\{1-[(1-(2-($ furan-2-ylmethylene)hydrazinyl)-4-methyl-1-oxopentan-2-yl)amino]-1-oxo-3phenylpropan-2-yl\}pyridine-3,5-dicarboxamide (6a):Yield 75\%, mp 205-207 ${ }^{\circ} \mathrm{C}$ (DMF/Ether). $[\alpha]_{\mathrm{D}}^{25}$ $=-105$ ( $c=0.5$, DMF). - IR (film): $v=3495-3350(\mathrm{NH}), 3095$ (CH-Ar), 2985 (CH-aliph.), 1654, 1532, $\left.1250\left(\mathrm{C}=\mathrm{O} \text {, amide I, II, and III) } \mathrm{cm}^{-1} .{ }^{1} \mathrm{H} \text { NMR spectrum (DMSO-d }\right)_{6}\right), \delta_{\mathrm{H}}, \mathrm{ppm}: 0.85-0.92(\mathrm{~m}, 12 \mathrm{H}$, $\left.4 \mathrm{CH}_{3}\right), 1.68-1.74\left(\mathrm{~m}, 4 \mathrm{H}, 2 \mathrm{CH}_{2}\right), 2.22-2.34(\mathrm{~m}, 2 \mathrm{H}, 2 \mathrm{CH}), 3.42\left(\mathrm{~d}, 4 \mathrm{H}, 2 \mathrm{CH}_{2}\right), 4.16-4.26(\mathrm{~m}, 2 \mathrm{H}, 2 \mathrm{CH})$, 4.45-4.58 (m, 2H, 2CH), 6.90-6.90 (m, 6H, 2 furyl ring), 7.12-7.38 (m, 10H, 2 Ph-H), $7.98(\mathrm{~s}, 2 \mathrm{H}, 2 \mathrm{CH}=\mathrm{N})$, $8.32,9.10\left(2 \mathrm{~s}, 3 \mathrm{H}\right.$, pyr-H) $, 8.78,8.80,9.16\left(3 \mathrm{~s}, 6 \mathrm{H}, 6 \mathrm{NH}\right.$, exchangeable with $\left.\mathrm{D}_{2} \mathrm{O}\right) .{ }^{13} \mathrm{C}$ NMR spectrum (DMSO-d 6$), \delta_{\mathrm{C}}$ ppm: 18.60, $19.15\left(4 \mathrm{C}, 4 \mathrm{CH}_{3}\right), 22.96(2 \mathrm{C}, 2 \mathrm{CH}), 40.25\left(2 \mathrm{C}, 2 \mathrm{CH}_{2}\right), 42.48\left(2 \mathrm{C}, \mathrm{CH}_{2}\right)$, 52.56, 53.34 (4C, 4 CH), 112.05, 117.95, 143.45, 148.16 (8C, 2 furyl-C), 125.84, 127.42, 127.98, 137.85 (12C, 2Ph-C), 131.62, 140.34, 152.40 (5C, pyr-C), 137.12 (2C, 2C=N), 163.95, 169.48 (4C, 4 CO-amide), 178.46 $(2 \mathrm{C}, 2 \mathrm{CO}-$ hydrazone). MS (EI, $70 \mathrm{eV}): \mathrm{m} / \mathrm{z}(\%)=872(16)[\mathrm{M}]^{+}$. Found, \%: C, 64.65; H, 6.10; N, 14.39 . $\mathrm{C}_{47} \mathrm{H}_{53} \mathrm{~N}_{9} \mathrm{O}_{8}$ (872.00). Calculated, \%: C, 64.74; $\mathrm{H}, 6.13 ; \mathrm{N}, 14.46$.

$\mathrm{N}^{3}, \mathrm{~N}^{5}$-bis\{1-[(4-methyl-1-oxo-1-(2-(thiophen-2-ylmethylene)hydrazinyl)pentan-2-yl)amino]-1-oxo3-phenylpropan-2-yl\}pyridine-3,5-dicarboxamide (6b). Yield 70\%, mp 218-220 ${ }^{\circ} \mathrm{C}$ (DMF/Ether). $[\alpha]_{\mathrm{D}}^{25}=-110$ ( $\left.c=0.5, \mathrm{DMF}\right)$. IR (film): $v=3482-3365(\mathrm{NH}), 3084(\mathrm{CH}-\mathrm{Ar}), 2978$ (CH-aliph.), 1655, 1535, $1254\left(\mathrm{C}=\mathrm{O}\right.$, amide I, II, and III) $\mathrm{cm}^{-1} .{ }^{1} \mathrm{H}$ NMR spectrum $\left(\right.$ DMSO- $\left._{6}\right), \delta_{\mathrm{H}}, \mathrm{ppm}: 0.82-0.90(\mathrm{~m}, 12 \mathrm{H}$, $\left.4 \mathrm{CH}_{3}\right), 1.65-1.70\left(\mathrm{~m}, 4 \mathrm{H}, 2 \mathrm{CH}_{2}\right), 2.20-2.30(\mathrm{~m}, 2 \mathrm{H}, 2 \mathrm{CH}), 3.44\left(\mathrm{~d}, 4 \mathrm{H}, 2 \mathrm{CH}_{2}\right), 4.18-4.30(\mathrm{~m}, 2 \mathrm{H}, 2 \mathrm{CH})$, $4.46-4.55(\mathrm{~m}, 2 \mathrm{H}, 2 \mathrm{CH}), 7.18-7.56(\mathrm{~m}, 16 \mathrm{H}, 2 \mathrm{Ph}-\mathrm{H}+2$ furyl ring), $7.86(\mathrm{~s}, 2 \mathrm{H}, 2 \mathrm{CH}=\mathrm{N}), 8.36,9.08(2 \mathrm{~s}$, $3 \mathrm{H}$, pyr-H) $, 8.75,8.84,9.18\left(3 \mathrm{~s}, 6 \mathrm{H}, 6 \mathrm{NH}\right.$, exchangeable with $\left.\mathrm{D}_{2} \mathrm{O}\right) .{ }^{13} \mathrm{C}$ NMR spectrum (DMSO-d 6 ), $\delta_{\mathrm{C}}$, ppm: 18.32, $19.10\left(4 \mathrm{C}, 4 \mathrm{CH}_{3}\right), 22.78(2 \mathrm{C}, 2 \mathrm{CH}), 40.55\left(2 \mathrm{C}, 2 \mathrm{CH}_{2}\right), 42.68\left(2 \mathrm{C}, \mathrm{CH}_{2}\right), 52.54,53.32$ $(4 \mathrm{C}, 4 \mathrm{CH}), 126.45,127.90,130.45,144.06$ (8C, 2 thienyl-C), 125.80, 127.52, 128.14, 137.80 (12C, 2Ph-C), 131.65, 140.36, 152.42 (5C, pyr-C), $125.18(2 \mathrm{C}, 2 \mathrm{C}=\mathrm{N}), 163.86,169.45$ (4C, 4 CO-amide), 178.42 (2C, 2CO-hydrazone). MS (EI, $70 \mathrm{eV}): \mathrm{m} / \mathrm{z}(\%)=904(24)[\mathrm{M}]^{+}$. Found, \%: C, 62.32; H, 5.86; N, 13.88; S, 7.00. $\mathrm{C}_{47} \mathrm{H}_{53} \mathrm{~N}_{9} \mathrm{O}_{6} \mathrm{~S}_{2}$ (904.12). Calculated, \%: C, 62.44; $\mathrm{H}, 5.91 ; \mathrm{N}, 13.94 ; \mathrm{S}, 7.09$.

\subsection{Biological Evaluation}

\subsubsection{In Vitro Cytotoxic Activity against MCF-7 Cancer Cells}

Human breast cancer (MCF-7) cells, obtained from Sigma-Aldrich Chemie GmbH, Taufkirchen, Germany, were propagated in RPMI-1640 supplemented with $10 \%$ heat inactivated FBS, $2 \mathrm{mM}$ L-glutamine, and $1 \%$ standard antibiotic solution. Cells were incubated in a $5 \% \mathrm{CO}_{2}$ humidified incubator at $37{ }^{\circ} \mathrm{C}$ and passaged bi-weekly. The in vitro anti-proliferative activity of the newly synthesized derivatives was assayed using the standard MTT technique [54-56].

The results were expressed as $\mathrm{IC}_{50}$. Experiments were repeated at least in triplicate, to obtain good reproducibility between replicate wells with standard errors below $10 \%$. Furthermore, the cytotoxic 
effects of the prepared derivatives were evaluated against normal nonmalignant cells, non-tumorigenic MCF-10A, in order to find out if the synthesized derivatives have toxicity against normal cells. Additionally, the results were compared with reference compounds (cisplatin and milaplatin) as positive controls.

\subsubsection{In Vivo Human Breast Cancer Xenograft}

The breast cancer xenograft model protocol was approved by the Institutional Animal Use and Care Committee of the University of Alabama at Birmingham (50-01-05-08B). Female athymic pathogen-free nude mice (nu/nu, 4-6 weeks) were used. Firstly, MCF-7 xenografts were initiated by implanting pellets slowly releasing estrogen for two months $(1.7 \mathrm{mg} 17 \beta$-estradiol/pellet) subcutaneously in the female nude mice. After $24 \mathrm{~h}$, confluent MCF-7 cells were harvested, washed two times with serum-free medium, re-suspended, and injected subcutaneously (s.c.) $\left(5 \times 10^{6}\right.$ cells, total volume $\left.0.2 \mathrm{~mL}\right)$ into the left inguinal area of the mice. Caliper measurement was used to measure tumor growth in two perpendicular diameters of the implant after $48 \mathrm{~h}$, and its volume was determined. Mice grafted with MCF-7 were divided into different groups (7-10 mice/group). Untreated mice received the solvent only. Treated groups received different prepared derivatives as previously described [57].

\subsubsection{In Vitro and In Vivo p53 Ubiquitination}

Different prepared derivatives 4-6 were evaluated for their inhibitory potential against p53 ubiquitination according to the previously reported procedure [57].

\subsubsection{LDHA Inhibition Assay}

Lactate dehydrogenase assay kit (Abcam, ab102526) and recombinant human LDHA protein (Abcam, ab93699) were used to assess the inhibitory effect of the tested compounds. First, we generated an NADH standard curve for colorimetric detection by measuring the OD (450 nm) at different molar amounts of NADH. The linear regression equation of the curve was derivatized in Graphpad Prism. Tested compounds were dissolved in DMSO and their inhibitory activity was assessed at 140 and $300 \mu \mathrm{M}$ strength. Galloflavin (Sigma, SML0776) at the same molar concentration was used as a known LDH inhibitor. The master reaction mix composed of LDH assay buffer and 10 ng human LDHA substrate per reaction was prepared. Tested compounds were added and the final reaction volume was adjusted to $50 \mu \mathrm{L}$. Absorbance at $450 \mathrm{~nm}$ was taken after 2-3 min (T-initial) and continued every minute for $118 \mathrm{~min}$ (T-final). The change in measurement over time (delta A450) was calculated as T-final minus T-initial. The amount of NADH generated by the assay between T-initial and T-final was deduced by comparing the A450 of each sample to the standard NADH standard curve. LDHA activity was calculated in milliunits per $\mathrm{mL}$ as the amount of NADH generated by the assay / (reaction time in minutes (118) $x$ reaction volume in $\mathrm{mL}(0.005)$ [45].

\subsection{Molecular Docking Studies}

The three-dimensional X-ray structure of LDHA (PDB code: 4ZVV) [53] was chosen as the template for the modeling study of the screened compounds 4-6. The crystal structure was derived from the RCSB Protein Data Bank and the molecular docking procedure was performed using MOE, 10.2008 software following the reported procedure [51,52].

\section{Conclusions}

In conclusion, a series of new tetrapeptide Schiff bases 3-6were designed, synthesized, and evaluated for their antitumor activities against breast MCF-7 cell lines. These compounds exhibited potent antiproliferative activities in comparison with the standards, cisplatin and milaplatin. All of these derivatives were subjected to evaluation ofin vitroandin vivosuppression of p53 ubiquitination and inhibition assay for LDHA enzyme. Molecular docking study was further performed to illustrate the 
possible binding interactions with LDHA that might be a promising base for the further development of novel anticancer agents with excellent LDH inhibitory activity.

Author Contributions: The listed authors contributed to this work as described in the following: A.E.-G.E.A. and R.E.A.M. gave the concepts of the work, interpreted the results, did the experimental part, and prepared the manuscript; M.E.-N. analyzed the data; E.S.N. and E.A.E. contributed to the anticancer activity and the modeling studies. All authors read and approved the final manuscript.

Acknowledgments: Authors are grateful to King Saud University for funding the work through Researchers Supporting Project (Project No. RSP-2019/66)".

Conflicts of Interest: The authors declare no conflict of interest.

\section{References}

1. Marqus, S.; Pirogova, E.; Piva, T.J. Evaluation of the use of therapeutic peptides for cancer treatment. J. Biomed. Sci. 2017, 24, 21. [CrossRef]

2. Chakrabarti, S.; Jahandideh, F.; Wu, J. Food-derived bioactive peptides on inflammation and oxidative stress. Biomed. Res. Int. 2014, 2014, 608979. [CrossRef] [PubMed]

3. Yang, R.Y.; Zhang, Z.F.; Pei, X.R.; Han, X.L.; Wang, J.B.; Wang, L.L.; Long, Z.; Shen, X.Y.; Li, Y. Immunomodulatory effects of marine oligopeptide preparation from Chum Salmon (Oncorhynchus keta) in mice. Food Chem. 2009, 113, 464-470. [CrossRef]

4. Cicero, A.F.G.; Fogacci, F.; Colletti, A. Potential role of bioactive peptides in prevention and treatment of chronic diseases: A narrative review. Br. J. Pharmacol. 2017, 174, 1378-1394. [CrossRef] [PubMed]

5. Holohan, C.; Van Schaeybroeck, S.; Longley, D.B.; Johnston, P.G. Cancer drug resistance: an evolving paradigm. Nat. Rev. Cancer 2013, 13, 714-726. [CrossRef] [PubMed]

6. Boohaker, R.J.; Lee, M.W.; Vishnubhotla, P.; Perez, J.M.; Khaled, A.R. The use of therapeutic peptides to target and to kill cancer cells. Curr. Med. Chem. 2012, 19, 3794-3804. [CrossRef]

7. Gautam, A.; Kapoor, P.; Chaudhary, K.; Kumar, R.; Raghava, G.P. Tumor homing peptides as molecular probes for cancer therapeutics, diagnostics and theranostics. Curr. Med. Chem. 2014, 21, 2367-2391. [CrossRef]

8. Vlieghe, P.; Lisowski, V.; Martinez, J.; Khrestchatisky, M. Synthetic therapeutic peptides: science and market. Drug Discov. Today 2010, 15, 40-56. [CrossRef]

9. Blanco-Míguez, A.; Gutiérrez-Jácome, A.; Pérez-Pérez, M.; Pérez-Rodríguez, G.; Catalán-García, S.; Fdez-Riverola, F.; Lourenço, A.; Sánchez, B. From amino acid sequence to bioactivity: scientific evidence on antitumor peptides. Protein Sci. 2016, 25, 1084-1095. [CrossRef]

10. Lopci, E.; Nanni, C.; Rampin, L.; Rubello, D.; Fanti, S. Clinical applications of 68Ga-1239 DOTANOC in neuroendocrine tumours. Minerva Endocrinol. 2008, 33, 277-281.

11. Emons, G.; Sindermann, H.; Engel, J.; Schally, A.V.; Grundker, C. Luteinizing hormone-releasing hormone receptor-targeted chemotherapy using AN-152. Neuroendocrinology 2009, 90, 15-18. [CrossRef] [PubMed]

12. Amr, A.E.; Naglah, A.M.; Sabry, N.M.; Ibrahim, A.A.; Elsayed, E.A.; Attar, A. Synthesis and investigation of 3,5-bis-linear and macrocyclic tripeptidopyridine candidates by using l-valine, N,N'-(3,5-pyridinediyldicarbonyl)bis-dimethyl ester as synthon. Z. Naturforsch. 2019, 74, 473-478. [CrossRef]

13. Khalifa, N.M.; Naglah, A.M.; Al-Omar, M.A.; Abo-Ghalia, M.H.; Amr, A.E. Synthesis and reactions of new chiral linear carboxamides with an incorporated peptide linkage using nalidixic acid and amino acids as starting materials. Z. Naturforsch. 2014, 69, 351-361. [CrossRef]

14. Khayyat, S.; Amr, A.E. Synthesis and biological activities of some new ( $\mathrm{N} \alpha$-dinicotinoyl)-bis- L-leucyllnear and macrocyclic peptides. Molecules 2014, 19, 10698-10716. [CrossRef] [PubMed]

15. Amr, A.E.; Abu Ghalia, M.H.; Al-Omar, M.A.; Abdalla, M.M. Anticancer activities of some macrocyclic peptidocalix[4]arene and peptidopyridine candidates. Lat. Am. J. Pharm. 2016, 35, 734-739.

16. Fahmi, N.; Shrivastava, S.; Meena, R.; Joshi, S.; Singh, R. Microwave assisted synthesis, spectroscopic characterization and biological aspects of some new chromium (III) complexes derived from NA O donor Schiff bases. N. J. Chem. 2013, 37, 1445-1453. [CrossRef]

17. Baseer, M.A.; Jadhav, V.D.; Phule, R.M.; Archana, Y.V.; Vibhute, Y.B. Synthesis and antibacterial activity of some new Schiff bases. Orient J. Chem. 2000, 16, 553-556.

18. Singh, W.M.; Dash, B.C. Synthesis of some new Schiff bases containing thiazole and oxazole nuclei and their fungicidal activity. Pesticides 1988, 22, 33-37. 
19. Sridhar, S.; Pandeya, S.; De Clercq, E. Synthesis and anti-HIV activity of some isatin derivatives. Boll. Chim. Farm. 2000, 140, 302-305.

20. Das, B.P.; Choudhury, T.R.; Das, G.K.; Chowdhury, D.N.; Choudhury, B. Comparative studies on largicidal activity of some Schiff bases with correspondian amines. Chem. Environ. Res. 1994, 3, 19-23.

21. Sparatore, F.; Pirisino, G.; Alamanni, M.; Manca-Dimich, P.; Satta, M. Azomethine derivatives with anti-inflammatory activity. Boll. Chim. Farm. 1978, 117, 638-651. [PubMed]

22. Chaviara, A.T.; Christidis, P.C.; Papageorgiou, A.; Chrysogelou, E.; Hadjipavlou-Litina, D.J.; Bolos, C.A. In vivo anticancer, anti-inflammatory, and toxicity studies of mixed-ligand $\mathrm{Cu}(\mathrm{II})$ complexes of dien and its Schiff dibases with heterocyclic aldehydes and 2-amino-2-thiazoline. Crystal structure of [Cu (dien)(Br)(2a-2tzn)](Br)(H(2)O). J. Inorg. Biochem. 2005, 99, 2102-2109. [CrossRef] [PubMed]

23. Jamshidvand, A.; Sahihi, M.; Mirkhani, V.; Moghadam, M.; Mohammadpoor-Baltork, I.; Tangestaninejad, S.; Rudbari, H.A.; Kargar, H.; Keshavarzi, R.; Gharaghani, S. Studies on DNA binding properties of new Schiff base ligands using spectroscopic, electrochemical and computationalmethods: Influence of substitutions on DNA-binding. J. Mol. Liq. 2018, 253, 61-71. [CrossRef]

24. Hameeda, A.; Al-Rashida, M.; Uroosc, M.; Ali, S.A.; Khan, K.M. Schiff bases in medicinal chemistry: A patent review (2010-2015). Expert. Opin. Ther. Pat. 2017, 27, 63-79. [CrossRef] [PubMed]

25. Di Stefano, G.; Manerba, M.; Di Ianni, L.; Fiume, L. Lactate dehydrogenase inhibition: Exploring possible applications beyond cancer treatment. Future Med. Chem. 2016, 8, 713-725. [CrossRef] [PubMed]

26. Warburg, O. On the origin of cancer cells. Science 1956, 123, 309-314. [CrossRef] [PubMed]

27. Talaiezadeh, A.; Shahriari, A.; Tabandeh, M.R.; Fathizadeh, P.; Mansouri, S. Kinetic characterization of lactate dehydrogenase in normal and malignant human breast tissues. Cancer Cell Int. 2015, 15, 19. [CrossRef]

28. Ward, R.A.; Brassington, C.; Breeze, A.L.; Caputo, A.; Critchlow, S.; Davies, G.; Goodwin, L.; Hassall, G.; Greenwood, R.; Holdgate, G.A.; et al. Design and synthesis of novel lactate dehydrogenase A inhibitors by fragment-based lead generation. J. Med. Chem. 2012, 55, 3285-3306. [CrossRef]

29. Kohlmann, A.; Zech, S.G.; Li, F.; Zhou, T.; Squillace, R.M.; Commodore, L.; Greenfield, M.T.; Lu, X.; Miller, D.P.; Huang, W.S.; et al. Fragment growing and linking lead to novel nanomolar lactate dehydrogenase inhibitors. J. Med. Chem. 2013, 56, 1023-1040. [CrossRef]

30. Altamimi, A.S.; Alafeefy, A.M.; Balode, A.; Vozny, I.; Pustenko, A.; El Shikh, M.E.; Alasmary, F.A.S.; Abdel-Gawad, S.A.; Žalubovskis, R. Symmetric molecules with 1,4-triazole moieties as potent inhibitors of tumour-associated lactate dehydrogenase-A. J. Enzy. Inh. Med. Chem. 2018, 33, 147-150. [CrossRef]

31. Amr, A.E.; Abo-Ghalia, M.H.; Abdalla, M.M. Synthesis of novel macrocyclic peptidocalix[4]arenes and peptido-pyridines as precursors for potential molecular metallacages, chemo-sensors and biologically active candidates. Z. Naturforsch. 2006, 61, 1335-1345.

32. Amr, A.E.; Abo-Ghalia, M.H.; Moustafa, G.O.; Al-Omar, M.A.; Nossier, E.S.; Elsayed, E.A. Synthesis and characterization of some newly macrocyclic pentapeptide derivatives as anticancer activity. Molecules 2018, 23, 2416. [CrossRef] [PubMed]

33. Amr, A.E.; Al-Omar, M.A.; Abdalla, M.M. Analgesic, anti-convulsant and antiparkinsonian activities of some synthesized 2,6-bis(tetracarbox-amide)-pyridine and macrocyclic tripeptide derivatives. Int. J. Pharmacol. 2016, 12, 74-80.

34. Khayyat, S.; Amr, A.E.; Abd El-Salam, O.I.; Al-Omar, M.A.; Abdalla, M.M. Analgesic and anti-inflammatory activities of some newly synthesized 3,5-bis[(peptidohydrazinyl)pyridine Schiff bases. Int. J. of Pharmacol. 2015, 11, 423-431. [CrossRef]

35. Flefel, E.M.; Alsafi, M.A.; Alahmadi, S.M.; Amr, A.E.; Fayed, A.A. Antimicrobial activities of some synthesized macrocyclic pentaazapyridine and dipeptide pyridine derivatives. Biomed. Res. 2018, 29, 1407-1413. [CrossRef]

36. Khalifa, N.M.; Amr, A.E.; Al-Omar, M.A.; Nossier, E.S. Synthesis, characterization, and antimicrobial activity of some chiral linear carboxamides with incorporated peptide linkage. Russ. J. General Chem. 2016, 86, 2785-2790. [CrossRef]

37. Azab, M.E.; Flefel, E.M.; Sabry, N.M.; Amr, A.E. Synthesis and antimicrobial activity of some linear dipeptide pyridine and macrocyclic pentaazapyridine candidates. Z. Naturforsch. 2016, 71, 803-810. [CrossRef]

38. Amr, A.E.; Ali, K.A.; Abdalla, M.M. Cytotoxic, antioxidant activities and structure activity relationship of some newly synthesized terpenoidaloxaliplatin analogs. Eur. J. Med. Chem. 2009, 44, 901-907. [CrossRef] 
39. Naglah, A.M.; Amr, A.E.; Abdel Mageid, R.E.; Al-Omar, M.A.; Abd El-Salam, O.I. Synthesis of chiral 3,5-bis(L-phenylalaninyl-L-leucinyl)pyridine Schiff base and their macrocyclic carboxaimide derivatives using 3,5-bis(L-phenylalaninyl)pyridine methyl ester. Z. Naturforsch. 2019. [CrossRef]

40. Abo-Ghalia, M.; Amr, A. Synthesis and investigation of a new cyclo ( $N^{\alpha}$-dipicolinoyl) pentapeptide of a breast and CNS cytotoxic activity and an ionophoric specificity. Amino Acids 2004, 26, 283-289. [CrossRef]

41. AbouMelha, K.S.A.; Al-Hazmi, G.A.A.; Refat, M.S. Synthesis of nano-metric gold complexes with new Schiff bases derived from 4-aminoantipyrene, their structures and anticancer activity. Russ. J. Gen. Chem. 2017, 87, 3043-3051. [CrossRef]

42. Padhye, S.; Yang, H.; Jamadar, A.; Cui, Q.C.; Chavan, D.; Dominiak, K.; McKinney, J.; Banerjee, S.; Dou, Q.P.; Sarkar, F.H. New difluoroKnoevenagel condensates of curcumin, their Schiff bases and copper complexes as proteasome inhibitors and apoptosis inducers in cancer cells. Pharm. Res. 2009, 26, 1874-1880. [CrossRef] [PubMed]

43. Dai, M.-S.; Lu, H. Inhibition of MDM2-mediated p53 Ubiquitination and Degradation by Ribosomal Protein L5. J. Biol. Chem. 2004, 279, 44475-44482. [CrossRef] [PubMed]

44. Manerba, M.; Vettraino, M.; Fiume, L.; Di Stefano, G.; Sartini, A.; Giacomini, E.; Buonfiglio, R.; Roberti, M.; Recanatini, M. Galloflavin (CAS 568-80-9): A novel inhibitor of lactate dehydrogenase. Chem. Med. Chem. 2012, 7, 311-317. [CrossRef] [PubMed]

45. Amr, A.E.; El-Shehry, M.F.; Ibrahim, A.A.; Hosni, H.M.; Al-Omar, M.A.; Ghabbour, H.A. Synthesis and molecular docking of new thiophene derivatives as lactate dehydrogenase-A inhibitors. Mini Rev. in Med. Chem. 2019, 19, 833-841. [CrossRef] [PubMed]

46. Farabegoli, F.; Vettraino, M.; Manerba, M.; Fiume, L.; Roberti, M.; Di Stefano, G. Galloflavin, a new lactate dehydrogenase inhibitor, induces the death of human breast cancer cells with different glycolytic attitude by affecting distinct signaling pathways. Eur. J. Pharm. Sci. 2012, 47, 729-738. [CrossRef] [PubMed]

47. Vettraino, M.; Manerba, M.; Govoni, M.; Di Stefano, G. Galloflavin suppresses lactate dehydrogenase activity and causes MYC downregulation in Burkitt lymphoma cells through NAD/NADH-dependent inhibition of sirtuin-1. Anticancer Drugs 2013, 24, 862-870. [CrossRef]

48. Manerba, M.; Di Ianni, L.; Fiume, L.; Roberti, M.; Recanatini, M.; Di Stefano, G. Lactate dehydrogenase inhibitors sensitize lymphoma cells to cisplatin without enhancing the drug effects on immortalized normal lymphocytes. Eur. J. Pharm. Sci. 2015, 74, 95-102. [CrossRef]

49. Han, X.; Sheng, X.; Jones, H.M.; Jackson, A.L.; Kilgore, J.; Stine, J.E.; Schointuch, M.N.; Zhou, C.; Bae-Jump, V.L. Evaluation of the anti-tumor effects of lactate dehydrogenase inhibitor galloflavin in endometrial cancer cells. J. Hematol. Oncol. 2015, 8, 2-8. [CrossRef]

50. Manerba, M.; Di Ianni, L.; Govoni, M. Lactate dehydrogenase inhibitors can reverse inflammation induced changes in colon cancer cells. Eur. J. Pharm. Sci. 2017, 96, 37-44. [CrossRef]

51. Elzahabi, H.S.A.; Nossier, E.S.; Khalifa, N.M.; Alasfoury, R.A.; El-Manawat, M.A. Anticancer evaluation and molecular modeling of multi-targeted kinase inhibitors based pyrido[2-d]pyrimidine scaffold. J. Enzy. Inh. Med. Chem. 2018, 33, 546-557. [CrossRef] [PubMed]

52. Othman, I.M.M.; Gad-Elkareem, M.A.M.; El-Naggar, M.; Nossier, E.S.; Amr, A.E. Novel phthalimide based analogues: Design, synthesis, biological evaluation, and molecular docking studies. J. Enzy. Inh. Med. Chem. 2019, 34, 1259-1270. [CrossRef] [PubMed]

53. Boudreau, A.; Purkey, H.E.; Hitz, A.; Robarge, K.; Peterson, D.; Labadie, S.; Kwong, M.; Hong, R.; Gao, M.; Del Nagro, C.; et al. Metabolic plasticity underpins innate and acquired resistance to LDHA inhibition. Nat. Chem. Biol. 2016, 12, 779-786. [CrossRef] [PubMed]

54. Elsayed, E.A.; Sharaf-Eldin, M.A.; El-Enshasy, H.A.; Wadaan, M. In vitroassessment of anticancer properties of Moringaperegrina essential seed oil on different cell lines. Pak. J. Zool. 2016, 48, 853-859.

55. Elsayed, E.A.; Farooq, M.; Dailin, D.; El-Enshasy, H.A.; Othman, N.Z.; Malek, R.; Danial, E.; Wadaan, M. In vitro and in vivo biological screening of kefiran polysaccharide produced by Lactobacillus kefiranofaciens. Biomed. Res. 2017, 28, 594-600.

56. Amr, A.E.; El-Naggar, M.; Al-Omar, M.A.; Elsayed, E.A.; Abdalla, M.M. In vitro and in vivo anti-breast cancer activities of some synthesized pyrazolinyl-estran-17-one candidates. Molecules 2018, 23, 1572. [CrossRef] [PubMed] 
57. Amr, A.E.; Elsayed, E.A.; Al-Omar, M.A.; BadrEldin, H.O.; Nossier, E.S.; Abdallah, M.M. Design, Synthesis, Anticancer Evaluation and Molecular Modeling of Novel Estrogen Derivatives. Molecules 2019, $24,416$. [CrossRef]

Sample Availability: Samples of the compounds are available from the authors.

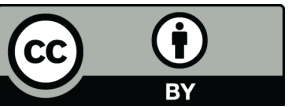

(C) 2020 by the authors. Licensee MDPI, Basel, Switzerland. This article is an open access article distributed under the terms and conditions of the Creative Commons Attribution (CC BY) license (http://creativecommons.org/licenses/by/4.0/). 